\title{
Quantum Theta Functions and Gabor Frames for Modulation Spaces
}

\author{
FRANZ LUEF ${ }^{1,2}$ and YURI I. MANIN ${ }^{3,4}$ \\ ${ }^{1}$ University of Vienna, Vienna, Austria.e-mail: franz.luef@univie.ac.at \\ ${ }^{2}$ University of California, Berkeley, USA. \\ ${ }^{3}$ Max-Planck-Institut für Mathematik, Bonn, Germany. \\ e-mail:manin@mpim-bonn.mpg.de \\ ${ }^{4}$ Northwestern University, Evanston, USA.
}

Received: 18 September 2008 / Revised: 11 February 2009 / Accepted: 11 February 2009 Published online: 26 February 2009 - (C) The Author(s) 2009. This article is published with open access at Springerlink.com

\begin{abstract}
Representations of the celebrated Heisenberg commutation relations in quantum mechanics (and their exponentiated versions) form the starting point for a number of basic constructions, both in mathematics and mathematical physics (geometric quantization, quantum tori, classical and quantum theta functions) and signal analysis (Gabor analysis). In this paper we will try to bridge the two communities, represented by the two co-authors: that of noncommutative geometry and that of signal analysis. After providing a brief comparative dictionary of the two languages, we will show, e.g. that the Janssen representation of Gabor frames with generalized Gaussians as Gabor atoms yields in a natural way quantum theta functions, and that the Rieffel scalar product and associativity relations underlie both the functional equations for quantum thetas and the Fundamental Identity of Gabor analysis.
\end{abstract}

Mathematics Subject Classification (2000). Primary 42C15，46L89; Secondary 11F27, $14 \mathrm{~K} 25$.

Keywords. quantum tori, Gabor frames, Weil representation, theta functions.

\section{Introduction}

Gabor analysis is a modern branch of signal analysis with various applications to pseudodifferential operators, harmonic analysis, function spaces, approximation theory, and quantum mechanics. It is well known that there are substantial connections between the mathematical foundations of signal analysis and those of quantum mechanics [21]. Furthermore, the theory of operator algebras furnished a rigorous framework for quantum mechanics, but possible direct relationships between signal analysis and operator algebras have not received much attention. Recent work of Gröchenig and his collaborators (see [25,27]) made explicit connections between the spectral invariance of certain Banach algebras and basic 
problems in signal analysis. In his $\mathrm{PhD}$ thesis [37] one of us has developed basic correspondences between Gabor analysis and noncommutative geometry over noncommutative tori.

Classical theta functions have a long history, see [47] for a modern exposition. From the functional theoretic viewpoint, they are holomorphic functions of several complex variables, which acquire an exponential factor after the shift by any vector in a period lattice. Geometrically, they represent sections of a line bundle over a complex torus, lifted to the universal cover of this torus and appropriately trivialized there.

The second named author, motivated by the ideas of geometric quantization, suggested in 1990 that one can develop a meaningful theory of quantum thetas after replacing ordinary complex tori in the classical construction by their quantum versions, see [42]. The new quantum theta functions were subsequently applied to the construction of algebraic quantization of abelian varieties in [43] (this case, as well as that of symplectic projective manifolds in general, presented a problem in Kontsevich's paper [35]) and to the program of Real Multiplication [45,64]. Thanks to the Boca study [2], it became clear that quantum thetas can be constructed as Rieffel's scalar products of vacuum vectors in representations of the appropriate Heisenberg groups. This idea, developed in [46], led to the discovery of a quantum version of those classical functional equations for theta functions that arise from different natural trivializations of a line bundle over a complex torus, see also [12].

In this paper we survey a new interpretation of quantum theta functions in the framework of Gabor analysis. Recent investigations have clarified and enriched parts of both subjects, see [40] for work related to Gabor frames, and [28] for a contribution on the structure of projective modules over noncommutative tori relying on methods from Gabor analysis. The present work is another instance for the relevance of Gabor analysis in exploiting basic notions of noncommutative geometry [7]. The basic link between Gabor analysis and noncommutative geometry is furnished by the Heisenberg group, especially the Schrödinger representation of the Heisenberg group. The Heisenberg group lies at the heart of various branches of physics, applied and pure mathematics, see the excellent survey [29]. After the groundbreaking work of Weil [65], theta functions have been linked with representations of Heisenberg groups. In this famous paper Weil introduced the metaplectic representation, which had independently been found by Shale. Weil's new methods and objects have influenced many mathematicians in their work on theta functions, most notably Cartier, Igusa and Reiter in [3,30,50-52]. In his work on abelian varieties Mumford had demonstrated the relevance of the Heisenberg group in the algebraization of theta functions, cf. [47]. In [61], Schempp has discussed the close relation between signal analysis and theta functions, where the Heisenberg group and its representation theory serves as a link between these two objects. The present investigation might be considered as a far-reaching extension of this line of research. 


\section{Central Extensions and Heisenberg Groups}

In this section we recall the basic definitions of central extensions and Heisenberg groups. Our presentation follows closely the one given in [46].

\subsection{CENTRAL EXTENSIONS}

Let $\mathcal{K}$ (resp. $\mathcal{Z}$ ) be an abelian group written additively (resp. multiplicatively). Consider a function $\psi: \mathcal{K} \times \mathcal{K} \rightarrow \mathcal{Z}$. Then the following conditions (a) and (b) are equivalent:

(a) $\psi(0,0)=1$ and $\psi$ is a cocycle, that is, for each $x, y, z$ we have

$$
\psi(x, y) \psi(x+y, z)=\psi(x, y+z) \psi(y, z) .
$$

(b) The following composition law on $\mathcal{G}:=\mathcal{Z} \times \mathcal{K}$ turns $\mathcal{G}=\mathcal{G}(\mathcal{K}, \psi)$ into a group with identity $(1,0)$ :

$$
(\lambda, x)(\mu, y):=(\lambda \mu \psi(x, y), x+y) .
$$

Moreover, if (a), (b) are satisfied, then the maps $\mathcal{Z} \rightarrow \mathcal{G}: \lambda \mapsto(\lambda, 0), \mathcal{G} \rightarrow \mathcal{K}$ : $(\lambda, x) \mapsto x$, describe $\mathcal{G}$ as a central extension of $\mathcal{K}$ by $\mathcal{Z}$ :

$$
1 \rightarrow \mathcal{Z} \rightarrow \mathcal{G}(\mathcal{K}, \psi) \rightarrow \mathcal{K} \rightarrow 1
$$

Notice that any bicharacter $\psi$ automatically satisfies (a). For arbitrary $\psi$, putting $x=0$ in (1.1), we see that $\psi(0, y)=1$ so that

$$
(\lambda, x)=(\lambda, 0)(1, x) .
$$

\subsubsection{Bicharacter $\varepsilon$}

Conversely, consider any central extension (1.3), choose a set theoretic section $\mathcal{K} \rightarrow$ $\mathcal{G}: x \mapsto \tilde{x}$ and define the map $\varepsilon: \mathcal{K} \times \mathcal{K} \rightarrow \mathcal{Z}$ by

$$
\varepsilon(x, y):=\tilde{x} \tilde{y} \tilde{x}^{-1} \tilde{y}^{-1} .
$$

Then $\varepsilon$ is a bicharacter which does not depend on the choice of a section and which is antisymmetric: $\varepsilon(y, x)=\varepsilon(x, y)^{-1}, \varepsilon(x, x)=1$. In particular, if $K \subset \mathcal{K}$ is a subgroup liftable to $\mathcal{G}$, then $K$ is $\varepsilon$-isotropic.

For the group $\mathcal{G}(\mathcal{K}, \psi)$, choosing $\tilde{x}=(1, x)$, we find

$$
\varepsilon(x, y)=\frac{\psi(x, y)}{\psi(y, x)},
$$

and if $\psi$ itself is an antisymmetric bicharacter, then $\varepsilon(x, y)=\psi(x, y)^{2}$. 


\subsubsection{Cohomological interpretation}

The class of $\psi$ in $H^{2}(\mathcal{K}, \mathcal{Z})$ determines $\mathcal{G}$ up to an isomorphism identical on $\mathcal{K}, \mathcal{Z}$. This extension is abelian iff $\varepsilon$ is trivial in which case the extensions are classified by elements of $\operatorname{Ext}^{1}(\mathcal{K}, \mathcal{Z})$. The map $\psi \mapsto \varepsilon$ coincides with the second arrow in the universal coefficients exact sequence

$$
\operatorname{Ext}^{1}(\mathcal{K}, \mathcal{Z}) \rightarrow H^{2}(\mathcal{K}, \mathcal{Z}) \rightarrow \operatorname{Hom}\left(\Lambda^{2} \mathcal{K}, \mathcal{Z}\right) .
$$

\subsection{REPRESENTATIONS OF CENTRAL EXTENSIONS}

Given $\mathcal{K}, \mathcal{Z}, \psi$ and a ground field $k$, choose in addition a character $\chi: \mathcal{Z} \rightarrow k^{*}$. Consider a linear space of functions $\mathbf{f}: \mathcal{K} \rightarrow k$ invariant with respect to the affine shifts and define operators $U_{(\lambda, x)}$ on this space by

$$
\left(U_{(\lambda, x)} \mathbf{f}\right)(x):=\chi(\lambda \psi(x, y)) \mathbf{f}(x+y) .
$$

A straightforward check shows that this is a representation of $\mathcal{G}(\mathcal{K}, \psi)$. However, it is generally reducible. Namely, suppose that there is an $\varepsilon$-isotropic subgroup $K_{0} \subset$ $\mathcal{K}$ liftable to $\mathcal{G}(\mathcal{K}, \psi)$. Let $\sigma: K_{0} \rightarrow \mathcal{G}(\mathcal{K}, \psi), \sigma(y)=(\gamma(y), y)$ be such a lift. Denote by $F\left(\mathcal{K} / / K_{0}\right)$ the subspace of functions satisfying the following condition:

$$
\forall x \in \mathcal{K}, \quad y \in K_{0}, \quad\left(U_{(\gamma(y), y)} \mathbf{f}\right)(x):=\chi(\varepsilon(x, y)) \mathbf{f}(x),
$$

or, equivalently,

$$
\forall x \in \mathcal{K}, \quad y \in K_{0}, \quad \mathbf{f}(x+y)=\chi\left(\gamma(y)^{-1} \psi(y, x)^{-1}\right) \mathbf{f}(x) .
$$

This subspace is invariant with respect to (1.6). If we choose for $K_{0}$ a maximal isotropic subgroup, then this provides a minimal subspace of this kind.

Formula (1.8) shows that if we know the value of $\mathbf{f}$ at a point $x_{0}$ of $\mathcal{K}$, it extends uniquely to the whole coset $x_{0}+K_{0}$, hence the notation $F\left(\mathcal{K} / / K_{0}\right)$ suggesting "twisted" functions on the coset space $\mathcal{K} / K_{0}$.

\subsection{LOCALLY COMPACT TOPOLOGICAL GROUPS}

The formalism briefly explained above is only an algebraic skeleton. In the category of $L C A b$ of locally compact abelian topological groups and continuous homomorphisms, with properly adjusted definitions, one can get a much more satisfying picture.

First of all, choose $\mathcal{Z}:=\mathbf{C}_{1}^{*}=\left\{z \in \mathbf{C}^{*}|| z \mid=1\right\}$. This is a dualizing object: for each $\mathcal{K}$ in $L C A b$ there exists the internal $\operatorname{Hom}(\mathcal{K}, \mathcal{Z})$ object, called the character group $\widehat{\mathcal{K}}$, and the map $\mathcal{K} \mapsto \widehat{\mathcal{K}}$ extends to the equivalence of categories $L C A b \rightarrow L C A b^{o p}$ (Pontryagin's duality).

Let now $\psi$ be a continuous cocycle $\mathcal{K} \times \mathcal{K} \rightarrow \mathcal{Z}$ so that $\varepsilon$ is a continuous bicharacter. Call the extension $\mathcal{G}(\mathcal{K}, \psi)$ a Heisenberg group, if the map $x \mapsto \varepsilon(x, *)$ 
identifies $\mathcal{K}$ with $\widehat{\mathcal{K}}$. In the case $\mathcal{K}=\mathbf{R}^{2 N}$, we call $\mathcal{G}\left(\mathbf{R}^{2 N}, \psi\right)$ a vector Heisenberg group.

Choose $k=\mathbf{C}$, and $\chi$ continuous. The formula (1.6) makes sense, e.g. for continuous functions $f$. Especially interesting, however, is the representation on $L_{2}(\mathcal{K})$ which makes sense because the operators (21.6) are unitary with respect to the squared norm $\int_{\mathcal{K}}|\mathbf{f}|^{2} \mathrm{~d} \mu_{\text {Haar }}$. Of course, square integrable functions cannot be evaluated at points, so that $\mathbf{f}(x+y)$ in (1.6) must be understood as the result of shifting $\mathbf{f}$ by $y \in \mathcal{K}$; similar precautions should be taken in the formula (1.8) defining now the space $L_{2}\left(\mathcal{K} / / K_{0}\right)$ where $K_{0}$ is a closed isotropic subgroup (it is then automatically liftable to a closed subgroup), and in many intermediate calculations. See Mumford's treatment on pp. 5-11 of [47] specially tailored for readers with algebraic geometric sensibilities.

We will call $L_{2}\left(\mathcal{K} / / K_{0}\right)$, for a maximal isotropic subgroup $K_{0} \subset \mathcal{K}$, a basic representation of the respective Heisenberg group.

The central fact of the representation theory of a Heisenberg group $\mathcal{G}(\mathcal{K}, \psi)$, $\mathcal{K} \in L C A b, \chi=i d$, is this:

THEOREM 1.3.1. (i) A basic representation of $\mathcal{G}(\mathcal{K}, \psi)$ is unitary and irreducible.

(ii) Any unitary representation of $\mathcal{G}(\mathcal{K}, \psi)$ whose restriction to the center is the multiplication by the identical character is isomorphic to the completed tensor product of $L_{2}\left(\mathcal{K} / / K_{0}\right)$ and a trivial representation. In particular, various basic representations are isomorphic.

If (i), (ii) are satisfied, then the maps $\mathcal{Z} \rightarrow \mathcal{G}: \lambda \mapsto(\lambda, 0), \mathcal{G} \rightarrow \mathcal{K}:(\lambda, x) \mapsto x$, describe $\mathcal{G}$ as a central extension of $\mathcal{K}$ by $\mathcal{Z}$ :

$$
1 \rightarrow \mathcal{Z} \rightarrow \mathcal{G}(\mathcal{K}, \psi) \rightarrow \mathcal{K} \rightarrow 1
$$

For a proof we refer the reader to [47].

\section{Calculus of Representations of Heisenberg Groups}

In this section we will recall some well-known facts about representations of Heisenberg groups. In the first part of this section we introduce the notions of matrix coefficients, integrability and square-integrablity of a representation on a Hilbert space. In the second part we will focus on the representations of vector Heisenberg groups.

\subsection{BASIC NOTIONS}

Let $\mathcal{K}$ be an object of $L C A b$ and $\mathcal{H}$ a Hilbert space. We consider a unitary strongly continuous irreducible representation $\rho$ of $\mathcal{K}$ on $\mathcal{H}$. For $\mathbf{f}, \mathbf{g} \in \mathcal{H}$ we call 
$S_{\mathbf{g}} \mathbf{f}(k)=\langle\mathbf{f}, \rho(k) \mathbf{g}\rangle$ a matrix coefficient of the representation $\rho$ on $\mathcal{H}$. If there exists a non-trivial $\mathbf{g}$ in $\mathcal{K}$ such that $S_{\mathbf{g}} \mathbf{g}$ is square integrable with respect to the Haar measure on $\mathcal{K}$, then the irreducible representation $\rho$ is called square integrable.

The irreducible representation $\rho$ is integrable if at least one matrix coefficient $S_{\mathbf{g}} \mathbf{g}$ is integrable for a non-trivial $\mathbf{g} \in \mathcal{H}$, such a $\mathbf{g}$ is then called an integrable element of the irreducible and integrable representation $\rho$. We denote by $\mathcal{A}_{1}(\mathcal{K})$ the set of all integrable elements $g \in \mathcal{H}$ and call it the space of admissible atoms.

The square integrability of $\rho$ implies the existence of orthogonality relations for matrix coefficients: for $\mathbf{f}_{1}, \mathbf{f}_{2}, \mathbf{g}_{1}, \mathbf{g}_{2}$ in $\mathcal{H}$ the following holds, see, e.g. [17]:

$$
\int_{\mathcal{K}} S_{\mathbf{g}_{1}} \mathbf{f}_{1}(k) \overline{S_{\mathbf{g}_{2}} \mathbf{f}_{2}(k)} \mathrm{d} k=\left\langle\mathbf{f}_{1}, \mathbf{f}_{2}\right\rangle \overline{\left\langle\mathbf{g}_{1}, \mathbf{g}_{2}\right\rangle}
$$

Among important consequences of this identity we note the existence of a reconstruction formula for functions in $\mathcal{H}$. Let $\mathbf{f}$ be in $\mathcal{H}$ and $\left\langle\mathbf{g}_{1}, \mathbf{g}_{2}\right\rangle \neq 0$. Then we have

$$
f=\left\langle\mathbf{g}_{1}, \mathbf{g}_{2}\right\rangle^{-1} \int_{\mathcal{K}} S_{\mathbf{g}_{1}} \mathbf{f}_{1}(k) \rho(k) \mathbf{g}_{2} \mathrm{~d} k .
$$

In the case of the Heisenberg group $\mathcal{G}(\mathcal{K}, \psi)$ this yields the most general version of the resolution of identity and the orthogonality relations are known as Moyal's identity. The space of admissible elements $\mathcal{A}_{1}(\mathcal{G}(\mathcal{K}, \psi))$ for the Heisenberg group $\mathcal{G}(\mathcal{K}, \psi)$ provides a realization of Feichtinger's algebra [14]. Since the space of integrable elements of $\mathcal{G}(\mathcal{K}, \psi)$ is another way of defining Feichtinger's algebra, see [17]. Feichtinger's algebra is an important Banach space in harmonic anlaysis, time-frequency analysis and Gabor analysis. Later we discuss Feichtinger's algebra and some of its weighted versions in the case of vector Heisenberg groups in more detail.

In our discussion of projective modules over quantum tori we also have to deal with smooth vectors of representations of a Heisenberg group $\mathcal{G}(\mathcal{K}, \psi)$, where $\mathcal{K}$ is an elementary locally compact abelian group. Recall that this means $\mathcal{K}$ is of the form vector space $\times$ torus $\times$ lattice $\times$ finite group. We denote the Lie algebra of the Heisenberg group by $L$. A vector $\mathbf{f} \in \mathcal{H}$ is called smooth if for any $X_{1}, \ldots, X_{n} \in$ $L$ the following expression makes sense

$$
\delta U_{X_{1}} \circ \ldots \delta U_{X_{n}}(\mathbf{f})
$$

where $\delta U_{X}(\mathbf{f})$ is defined as the limit when $t \rightarrow 0$

$$
\delta U_{X}(\mathbf{f}):=\lim \frac{U_{\exp (t X)} \mathbf{f}-\mathbf{f}}{t} .
$$

It is known that the space $\mathcal{H}_{\infty}$ of smooth vectors is dense and the operators $\delta U_{X}$ are skew adjoint but unbounded [47]. 


\subsection{BASIC REPRESENTATIONS OF VECTOR HEISENBERG GROUPS}

Now we discuss the vector Heisenberg group $\mathcal{G}\left(\mathbf{R}^{2 N}, \psi\right)$ in more detail. Note that in this case we can choose $\psi$ as an antisymmetric bicharacter with values in $\mathbf{C}_{1}^{*}$. After choosing an appropriate basis, we identify $\mathbf{R}^{2 N}$ with the space of pairs of column vectors $x=\left(x_{1}, x_{2}\right), x_{i} \in \mathbf{R}^{N}$. In this case $\psi$ can be written in the form

$$
\psi_{A}(x, y)=\mathrm{e}^{\pi i A(x, y)}
$$

where $A: \mathcal{K} \times \mathcal{K} \rightarrow \mathbf{R}$ is a nondegenerate antisymmetric pairing, such as

$$
A(x, y)=x_{1}^{t} y_{2}-x_{2}^{t} y_{1}
$$

where $x_{i}^{t}$ denotes the transposed row vector. We have then $\varepsilon_{A}(x, y)=\mathrm{e}^{2 \pi i A(x, y)}$.

In particular, the subspace $\mathbf{R}^{N} \times\{0\}$ is a maximal $\varepsilon$-isotropic closed subgroup of $\mathbf{R}^{2 N}$.

We will recall the structure of two Heisenberg representations of $\mathcal{G}\left(\mathbf{R}^{2 N}, \psi_{A}\right)$ using normalizations adopted in [47].

Model I. In this model the unitary representation of $\mathcal{G}\left(\mathbf{R}^{2 N}, \psi\right)$ lives on $L_{2}\left(\mathbf{R}^{N}\right)$, the Hilbert space of square integrable complex functions $\mathbf{f}$ on $\mathbf{R}^{N}$ with the scalar product

$$
\langle\mathbf{f}, \mathbf{g}\rangle:=\int \mathbf{f}\left(x_{1}\right) \overline{\mathbf{g}\left(x_{1}\right)} \mathrm{d} \mu
$$

where $\mathrm{d} \mu$ is the Haar measure in which $\mathbf{Z}^{N}$ has covolume 1 .

The action of $\mathcal{G}\left(\mathbf{R}^{N}, \psi_{A}\right)$, with central character $\chi(\lambda)=\lambda$, is given by the formula

$$
\left(U_{(\lambda, y)} \mathbf{f}\right)\left(x_{1}\right)=\lambda \mathrm{e}^{2 \pi i x_{1}^{t} y_{2}+\pi i y_{1}^{t} y_{2}} \mathbf{f}\left(x_{1}+y_{1}\right) .
$$

Many authors refer to the Model I as the Schrödinger representation of $\mathcal{G}\left(\mathbf{R}^{2 N}, \psi_{A}\right)$.

Model $I I_{T}$. The second model is actually a family of models depending on the choice of a compatible Kähler structure upon $\mathcal{K}=\mathbf{R}^{2 N}$. A general Kähler structure on $\mathbf{R}^{2 N}$ can be given by a pair consisting of a complex structure and a Hermitean scalar product $H$. We will call this Kähler structure compatible (with the choice (2.4)) if $\operatorname{Im} H=A$. Such structures are parametrized by the Siegel space $\mathcal{S}_{N}$ consisting of symmetric matrices $T \in M(N, \mathbf{C})$ with positive definite $\operatorname{Im} T$.

In particular, the complex structure defined by $T$ identifies $\mathbf{R}^{2 N}$ with $\mathbf{C}^{N}$ via

$$
\left(x_{1}, x_{2}\right)=x \mapsto \underline{x}:=T x_{1}+x_{2},
$$

and we have

$$
H_{T}(\underline{x}, \underline{x})=\underline{x}^{t}(\operatorname{Im} T)^{-1} \underline{x}^{*}
$$

where $*$ denotes the componentwise complex conjugation. 
Consider the Hilbert space $\mathcal{H}_{T}$ of holomorphic functions on $\mathbf{C}^{N}=\mathcal{K}$ consisting of the functions with finite norm with respect to the scalar product

$$
\langle\mathbf{f}, \mathbf{g}\rangle_{T}:=\int_{\mathbf{C}^{N}} \mathbf{f}(\underline{x}) \overline{\mathbf{g}(\underline{x})} \mathrm{e}^{-\pi H_{T}(\underline{x}, \underline{x})} \mathrm{d} v
$$

where $\mathrm{d} v$ is the translation invariant measure making $\mathbf{Z}^{2 N}$ a lattice of covolume 1 in $\mathbf{R}^{2 N}$.

For $(\lambda, y) \in \mathcal{G}\left(\mathbf{C}^{N}, \psi_{A}\right)$ and a holomorphic function $f$ on $\mathbf{C}^{N}$, put

$$
\left(U_{(\lambda, y)}^{\prime} \mathbf{f}\right)(\underline{x}):=\lambda^{-1} \mathrm{e}^{-\pi H_{T}(\underline{x}, \underline{y})-\frac{\pi}{2} H_{T}(\underline{y}, \underline{y})} \mathbf{f}(\underline{x}+\underline{y}) .
$$

A straightforward check shows that these operators are unitary with respect to (2.9), and moreover, that they define a representation of $\mathcal{G}\left(\mathbf{C}^{N}, \psi_{A}\right)$ in $\mathcal{H}_{T}$ corresponding to the character $\chi(\lambda)=\lambda^{-1}$ of $\mathbf{C}_{1}^{*}$, in the sense of formula (1.8). This is generalization of the classical Bargmann-Fock representation, which corresponds to the choice $T=\tau I$ for $\tau$ with a positive imaginary part.

It turns out that this representation is irreducible on $\mathcal{H}_{T}$ and thus is a model of the Heisenberg representation.

The proof of irreducibility spelled out in [47] involves constructing vacuum vectors in $\mathcal{H}_{T}$ which in this model turn out to be simply constant functions. Translated via canonical (antilinear) isomorphism into other models they look differently. For example they become (proportional to) a "quadratic exponent" $\mathbf{f}_{T}:=$ $\mathrm{e}^{\pi i x_{1}^{t} T x_{1}}$ in Model I (i.e. $\left.L_{2}\left(\mathbf{R}^{2 N} / / \mathbf{R}^{N}\right)\right)$ or to an essentially classical theta-function

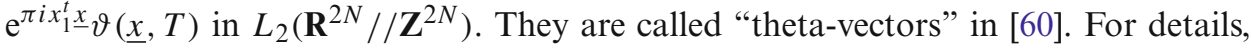
see the Theorem 2.2 in [47] and the discussion around it, see also [4].

\section{Principle Notions of Time-Frequency Analysis in the Context of Vector Heisenberg Groups}

In this section we interpret matrix coefficients of the Schrödinger representation of the vector Heisenberg group in terms of time-frequency analysis. This leads us naturally to the study of time-frequency representations, namely the short-time Fourier transform and the Wigner distribution from the phase space approach to quantum mechanics.

\subsection{TIME-FREQUENCY REPRESENTATIONS}

The basic task in signal analysis is to analyse the spectral content of a given signal f, i.e. a complex-valued function $\mathbf{f}(t)$ on $\mathbf{R}^{N}$. Traditionally one invokes the Fourier transform $\mathcal{F} \mathbf{f}$ to gain some insight about the spectral resolution of $\mathbf{f}$, i.e. the frequencies $\omega$ contained in the given signal $\mathbf{f}$. We define the Fourier transform $\mathcal{F} \mathbf{f}$ for 
$\mathbf{f} \in L_{1}\left(\mathbf{R}^{N}\right)$ by

$$
\mathcal{F} \mathbf{f}(\omega)=\widehat{\mathbf{f}}(\omega)=\int_{\mathbf{R}^{N}} \mathbf{f}(t) \mathrm{e}^{-2 \pi i t \cdot \omega} \mathrm{d} t .
$$

One drawback of this approach is that $\mathcal{F} \mathbf{f}$ does not provide any local information about the signal $\mathbf{f}$. Therefore one is looking for joint time and frequency representations. In time-frequency analysis it is very useful to consider $\mathbf{R}^{2 N}$ as

$$
\mathbf{R}^{\mathbf{2 N}}=\mathbf{R}^{N} \times \widehat{\mathbf{R}}^{N}=\left\{(t, \omega) \mid t, \omega \in \mathbf{R}^{N}\right\} .
$$

If we want to emphasize this description of $\mathbf{R}^{2 N}$ we refer to it as time-frequency space.

In order to obtain information about the "local frequency spectrum" of $\mathbf{f}$, we use a window $\mathbf{g}$ to localize the signal $\mathbf{f}$ and take the Fourier transform of this localized piece of $\mathbf{f}$.

Short-Time Fourier Transform (STFT) of a signal $\mathbf{f}$ with respect to a window function $\mathbf{g}$ :

$$
V_{\mathbf{g}} \mathbf{f}(x, \omega):=\int_{\mathbf{R}^{N}} \mathbf{f}(t) \overline{\mathbf{g}}(t-x) \mathrm{e}^{-2 \pi i t \cdot \omega} \mathrm{d} t .
$$

The properties of $V_{\mathbf{g}} \mathbf{f}$ depend crucially on the choice of the window $\mathbf{g}$. It turns out that the integrable and smooth vectors of the vector Heisenberg group provide good classes of window functions. If $\mathbf{f}$ and $\mathbf{g}$ are for example Schwartz functions on $\mathbf{R}^{N}$, then $V_{\mathbf{g}} \mathbf{f}(x, \omega)$ measures the amplitude of the frequency band near $\omega$ at time $x$.

Among the many facts about STFT, we want to mention one on the relation between the STFT of $\mathbf{f}$ and the STFT of $\widehat{f}$. By an application of Parseval's theorem to $V_{\mathbf{g}} \mathbf{f}$ one obtains

$$
V_{\widehat{\mathbf{g}}} \widehat{\mathbf{f}}(x, \omega)=\mathrm{e}^{-2 \pi i x \cdot \omega} V_{\mathbf{g}} \mathbf{f}(\omega,-x) .
$$

Therefore if we choose the Gaussian $g_{0}(t)=\mathrm{e}^{-\pi t^{2}}$ as a window function (or any window invariant under Fourier transform), then the time-frequency content of $\widehat{\mathbf{f}}$ is just the one of $\mathbf{f}$ rotated by an angle of $\pi / 2$.

During the last two decades a representation theoretic interpretation of the STFT has become of great relevance in time-frequency analysis, because it has allowed to put the time-frequency analysis on solid mathematical ground. The main proponents of this line of research are Feichtinger and Gröchenig, who described in [17] a correct framework for a rigorous treatment of time-frequency analysis, the coorbit theory. Later we briefly discuss some aspects of their coorbit theory for the Schrödinger representation of vector Heisenberg groups.

In time-frequency analysis one associates to a point $(x, \omega)$ in the time-frequency plane a time-frequency shift: the unitary operator $\pi(x, \omega)$ on $L_{2}\left(\mathbf{R}^{N}\right)$ defined by

$$
\mathbf{f} \mapsto \pi(x, \omega) \mathbf{f}(t):=\mathrm{e}^{2 \pi i t \cdot \omega} \mathbf{f}(t-x) .
$$


The same operator occurs in the basic representation of the central extension of the time-frequency space:

$$
\pi(x, \omega)=U_{\left(\mathrm{e}^{-\pi i t \cdot \omega},(x, \omega)\right)} \cdot
$$

The restriction of $\pi(x, \omega)$ to the maximal $\varepsilon_{A}$-isotropic subspaces $\mathbf{R}^{N} \times\{0\}$ and $\{0\} \times \mathbf{R}^{N}$ of the time-frequency space $\mathbf{R}^{2 N}$ are respectively the translation operator $T_{x}:=\pi(x, 0)$ and the modulation operator $M_{\omega}:=\pi(0, \omega)$.

The mapping $(x, \omega) \mapsto \pi(x, \omega)$ is a unitary (projective) strongly continuous representation of $\mathbf{R}^{2 N}$ on $L_{2}\left(\mathbf{R}^{N}\right)$. Therefore, we can express $V_{\mathbf{g}} \mathbf{f}$ as the matrixcoefficient of this projective representation, i.e.

$$
V_{\mathbf{g}} \mathbf{f}(x, \omega)=\langle\mathbf{f}, \pi(x, \omega) \mathbf{g}\rangle .
$$

This intrinsic description of STFT in terms of the Heisenberg group amplifies the great relevance of the STFT and makes it a basic object of study. Moreover, most other time-frequency representations, such as cross-Wigner distribution, have a description in terms of the STFT.

Recall, that the cross-Wigner distribution of two signals $\mathbf{f}$ and $\mathbf{g}$ is defined by

$$
W(\mathbf{f}, \mathbf{g})(x, \omega)=2^{N} \mathrm{e}^{4 \pi i x \cdot \omega} \int_{\mathbf{R}^{N}} \mathbf{f}\left(x+\frac{t}{2}\right) \overline{\mathbf{g}}\left(x-\frac{t}{2}\right) \mathrm{e}^{-2 \pi i t \cdot \omega} \mathrm{d} t .
$$

The cross-Wigner distribution is just a short-time Fourier transform in disguise:

$$
W(\mathbf{f}, \mathbf{g})(x, \omega)=2^{N} \mathrm{e}^{4 \pi i x \cdot \omega} V_{\tilde{\mathbf{g}}} \mathbf{f}(2 x, 2 \omega),
$$

where $\tilde{\mathbf{g}}(x)=\mathbf{g}(-x)$ denotes the reflection of $\mathbf{g}$ with respect to the origin.

\subsection{FUNCTION SPACES FOR TIME-FREQUENCY ANALYSIS}

Since STFT is one of the key players in the time-frequency analysis, it is natural to consider function spaces defined in terms of integrability conditions or decay conditions of the STFT. In the early 1980s, H. G. Feichtinger introduced the socalled modulation spaces in exactly this manner [15]. For a thorough discussion of the history and the relevance of modulation spaces in various branches of mathematics and engineering see the excellent survey article by Feichtinger [16]. Among the class of modulation spaces one Banach space, Feichtinger's algebra $S_{0}\left(\mathbf{R}^{N}\right)$, stands out as the most prominent member, already introduced by different methods in [14]. Feichtinger's algebra is a good substitute for the Schwartz class as long as one is not dealing with partial differential equations, see [19]. Feichtinger's algebra $S_{0}\left(\mathbf{R}^{N}\right)$ has an intrinsic description in terms of the Heisenberg group, namely it is the space of integrable vectors for the Schrödinger representation of the Heisenberg group $\mathcal{G}\left(\mathbf{R}^{2 N}, \psi_{A}\right)$.

Let $v_{s}$ be the submultiplicative weight $v_{s}(x, \omega)=\left(1+\|x\|_{2}^{2}+\|\omega\|_{2}^{2}\right)^{s / 2}$ on $\mathbf{R}^{2 N}$, i.e. $v_{s}(x+y, \omega+\eta) \leq v_{s}(x, \omega) v_{s}(y, \eta)$ for $(x, \omega),(y, \eta) \in \mathbf{R}^{2 N}$. Recall that a weight $m$ is 
called $v_{s}$-moderate, if $m(x+y, \omega+\eta) \leq v_{s}(x, \omega) m(y, \eta)$ for all $(x, \omega),(y, \eta) \in \mathbf{R}^{2 N}$. Let $g$ be a nonzero function in the Schwartz class $\mathcal{S}\left(\mathbf{R}^{N}\right)$. Then a tempered distribution $\mathbf{f} \in \mathcal{S}^{\prime}\left(\mathbf{R}^{N}\right)$ belongs to the modulation space $M_{p, q}^{m}\left(\mathbf{R}^{N}\right)$, if

$$
\|\mathbf{f}\|_{M_{p, q}^{m}}:=\left(\int_{\mathbf{R}^{N}}\left(\int_{\mathbf{R}^{N}}\left|V_{\mathbf{g}} \mathbf{f}(x, \omega)\right|^{p} m(x, \omega)^{p}\right)^{q / p} \mathrm{~d} \omega\right)^{1 / q}<\infty .
$$

For $1 \leq p, q \leq \infty$ the modulation spaces $M_{p, q}^{m}\left(\mathbf{R}^{N}\right)$ are Banach spaces, and different functions $\mathbf{g}$ yield equivalent norms on $M_{p, q}^{m}\left(\mathbf{R}^{N}\right)$. We will write $M_{p}$ for $M_{p, p}$. The properties of modulation spaces are by their definition related to the properties of the short-time Fourier transform.

The modulation spaces $M_{1}^{v_{s}}\left(\mathbf{R}^{N}\right)$ are of great relevance in Gabor analysis, since they provide a natural class of window functions. We denote $M_{1}^{v_{s}}\left(\mathbf{R}^{N}\right)$ by $M_{1}^{s}\left(\mathbf{R}^{N}\right)$. The space $M_{1}\left(\mathbf{R}^{N}\right)=M_{1}^{v_{0}}\left(\mathbf{R}^{N}\right)$ is the well-known Feichtinger algebra $S_{0}\left(\mathbf{R}^{N}\right)$ [16].

Below we summarize some properties used later in our treatment of projective modules over quantum tori.

PROPOSITION 3.2.1. The following holds:

(i) $M_{p, q}^{m}\left(\mathbf{R}^{N}\right)$ is invariant under time-frequency shifts, i.e.

$$
\|\pi(y, \eta) \mathbf{f}\|_{M_{p, q}^{m}} \leq C v_{s}(y, \eta)\|\mathbf{f}\|_{M_{p, q}^{m}} .
$$

(ii) $M_{p, q}^{m}\left(\mathbf{R}^{N}\right)$ is invariant under Fourier transform, i.e.

$$
\|\widehat{\mathbf{f}}\|_{M_{p, q}^{m}} \leq C\|\mathbf{f}\|_{M_{p, q}^{m}} .
$$

(iii) Let $\mathbf{f}, \mathbf{g}$ be in $M_{1}^{s}\left(\mathbf{R}^{N}\right)$. Then $V_{\mathbf{g}} \mathbf{f}$ is in $L_{1}\left(\mathbf{R}^{N}\right)$.

For proofs of these statements we refer to [26] or [20]. The basic strategy for proving (i) and (ii) is to establish these properties for STFT. The statement (i) follows from the covariance property of STFT:

$$
V_{\mathbf{g}}(\pi(y, \eta) \mathbf{f})(x, \omega)=\mathrm{e}^{2 \pi i y \cdot \omega} V_{\mathbf{g}} \mathbf{f}(x-y, \omega-\eta) .
$$

The proof of (ii) relies on (3.7).

Now we want to give a global description of the smooth vectors $\mathcal{H}_{\infty}$ for the vector Heisenberg group $\mathcal{G}\left(\mathbf{R}^{N}, \psi_{A}\right)$. In this case $\mathcal{H}_{\infty}$ is the space of Schwartz functions $\mathcal{S}\left(\mathbf{R}^{N}\right)$. The basic observation is that

$$
\mathcal{S}\left(\mathbf{R}^{N}\right)=\bigcap_{s \geq 0} M_{1}^{s}\left(\mathbf{R}^{N}\right) \text {. }
$$

Consequently, $f \in \mathcal{S}\left(\mathbf{R}^{N}\right)$ if and only if $\|\mathbf{f}\|_{M_{1}^{s}}$ is finite for all $s \geq 0$, see [26] for a proof. Note that this description of $\mathcal{S}\left(\mathbf{R}^{N}\right)$ allows one to transfer many statements from $M_{1}^{S}\left(\mathbf{R}^{N}\right)$ to $\mathcal{S}\left(\mathbf{R}^{N}\right)$. 
We want to close this section with a discussion of the symplectic Fourier transform. For $\mathbf{F} \in L_{1}\left(\mathbf{R}^{2 N}\right)$ we define its symplectic Fourier transform by

$$
\mathcal{F}_{S} \mathbf{F}(x, \omega)=\int_{\mathbf{R}^{2 N}} \mathbf{F}(y, \eta) \mathrm{e}^{2 \pi i(y \cdot \omega-x \cdot \eta)} d y d \eta .
$$

Observe that for a fixed $(x, \omega) \in \mathbf{R}^{2 N}$ the set $\left\{\mathrm{e}^{2 \pi i(y \cdot \omega-x \cdot \eta)} \mid(y, \eta) \in \mathbf{R}^{2 N}\right\}$ is actually the character group of the time-frequency plane, i.e. $\widehat{\mathbf{R}}^{N} \times \mathbf{R}^{N}$. Therefore the symplectic Fourier transform is the ordinary Fourier transform of the timefrequency plane $\mathbf{R}^{N} \times \widehat{\mathbf{R}}^{N}$.

As in the Euclidan case, $\mathbf{M}_{\mathbf{1}}{ }^{v_{s} \otimes v_{s}}\left(\mathbf{R}^{2 N}\right)$ is invariant under the symplectic Fourier transform and consequently $\mathcal{S}\left(\mathbf{R}^{2 N}\right)$ is invariant under the symplectic Fourier transform. The following fact has important consequences in Gabor analysis (Janssen representation of a Gabor frame operator, see [19,20,34]) and in the construction of projective modules over quantum tori (see [53-56]).

PROPOSITION 3.2.2. Let $\mathbf{f}_{1}, \mathbf{f}_{2}, \mathbf{g}_{1}, \mathbf{g}_{2}$ be in $M_{1}^{s}\left(\mathbf{R}^{N}\right)$. Then

$$
\left.\left.\mathcal{F}_{S}\left(V_{\mathbf{g}_{1}} \mathbf{f}_{1}\right) \cdot \overline{\left(V_{\mathbf{g}_{2}} \mathbf{f}_{2}\right.}\right)(y, \eta)=\overline{\left(V_{\mathbf{f}_{1}} \mathbf{f}_{2}\right.} \cdot V_{\mathbf{g}_{2}} \mathbf{g}_{1}\right)(y, \eta) .
$$

In [13] the authors point out that this identity has been known in the signal analysis community since the early 1960's, when Sussman obtained this fact in his work on time-varying filters [63]. Later Rieffel gave a proof for signals and windows in $\mathcal{S}\left(\mathbf{R}^{N}\right)$ in [53-56], which follows from the statement as indicated above. In Gabor analysis Feichtinger and Kozek were the first who explicitly formulated this fact for Feichtinger's algebra $S_{0}\left(\mathbf{R}^{N}\right)$. In [20] we have discussed this identity in great detail for signals in dual classes of modulation spaces. The main idea is to choose the signals in such a way that the product of the STFT's is in $M_{1}\left(\mathbf{R}^{2 N}\right)$ and then use its invariance under the symplectic Fourier transform to justify the application of the symplectic Fourier transform.

\section{Quantum Tori Associated to Embedded Lattices in the Vector Heisenberg Groups}

We briefly recall the notions of quantum theta functions, Heisenberg group of quantum tori and quantum tori associated to embedded lattices. Our presentation follows largely the lines of [46].

\subsection{HEISENBERG GROUP OF QUANTUM TORUS AND QUANTUM THETA FUNCTIONS}

Let $H$ be a free abelian group of finite rank written additively, $k$ a ground field, and $\alpha: H \times H \rightarrow k^{*}$ a skewsymmetric pairing. The quantum torus $T(H, \alpha)$ with the character group $H$ and quantization parameter $\alpha$ is represented by an algebra 
generated by a family of formal exponents $\left\{e(h)=e_{H, \alpha}(h), h \in H\right\}$, satisfying the relations

$$
e(g) e(h)=\alpha(g, h) e(g+h) .
$$

In particular, $T(H, 1)$ is an algebraic torus, spectrum of the group algebra $k[e(h) \mid h \in H]$ of $H$. The group of its points $x \in T(H, 1)(k)=\operatorname{Hom}\left(H, k^{*}\right)$ acts upon functions on $T(H, \alpha)$ mapping $e_{H, \alpha}(h)$ to $x^{*}\left(e_{H, \alpha}(h)\right):=h(x) e_{H, \alpha}(h)$ where $h(x)$ denotes the value of the character $e(h)$ at $x$.

The Heisenberg group of the quantum torus $T(H, \alpha)$ introduced in [44] and denoted there $\mathcal{G}(H, \alpha)$ consists of all maps of the form

$$
\Phi \mapsto c e_{H, \alpha}(g) x^{*}(\Phi) e_{H, \alpha}(h)^{-1}, c \in k^{*} ; x \in T(H, 1)(k) ; g, h \in H,
$$

where $\Phi$ is a formal theta function, see [44, Eq. (0.19)]. Any such map has a unique representative of the same form in which $h=0$ ("left representative"). Writing this representative as $[c ; x, g]$ we get the composition law

$$
\left[c^{\prime} ; x^{\prime}, g^{\prime}\right][c ; x, g]=\left[c^{\prime} c g\left(x^{\prime}\right) \alpha\left(g^{\prime}, g\right) ; x^{\prime} x, g^{\prime}+g\right] \text {. }
$$

In other words, this group is the central extension of $\operatorname{Hom}\left(H, k^{*}\right) \times H$ by $k^{*}$ corresponding to the bicharacter

$$
\psi\left(\left(x^{\prime}, g^{\prime}\right),(x, g)\right)=g\left(x^{\prime}\right) \alpha\left(g^{\prime}, g\right)
$$

and having the associated bicharacter

$$
\varepsilon\left(\left(x^{\prime}, g^{\prime}\right),(x, g)\right)=g\left(x^{\prime}\right) g^{\prime}(x)^{-1} \alpha^{2}\left(g^{\prime}, g\right) .
$$

In particular, if a subgroup $B \subset \operatorname{Hom}\left(H, k^{*}\right) \times H$ is liftable to $\mathcal{G}(H, \alpha)$, the form (4.4) restricted to $B$ must be symmetric: this is the main part of Lemma 2.2 in [44].

A lift $\mathcal{L}$ of $B$ to a subgroup of $\mathcal{G}(H, \alpha)$ is called a multiplier. The restriction to $B$ of the form (4.4), $\langle\rangle:, B \times B \rightarrow k^{*}$, is called the structure form of this multiplier. (Formal) linear combinations of the exponents $e_{H, \alpha}$ invariant with respect to the action of $\mathcal{L}(B)$ constitute a linear space $\Gamma(\mathcal{L})$ and are called (formal) quantum theta functions.

\subsection{EMBEDDED LATTICES AND QUANTUM TORI}

In this section $\mathcal{K}$ denotes an object of $L C A b, \psi$ is a bicharacter of $\mathcal{K}$ such that $\varepsilon$ (cf. (1.5)) identifies $\mathcal{K}$ with $\widehat{\mathcal{K}}$. Let $\mathcal{G}(\mathcal{K}, \psi)$ be the respective Heisenberg group, central extension of $\mathcal{K}$ by $\mathcal{Z}=\mathbf{C}_{1}^{*}$ as above.

We will call an embedded lattice a closed subgroup $D \subset \mathcal{K}$ such that $D$ is a finitely generated free abelian group, whereas $\mathcal{K} / D$ is a topological torus. In this section we consider only those groups $\mathcal{K}$ which admit embedded lattices. 
Consider a family of constants

$$
\left\{c_{h} \in \mathbf{C}_{1}^{*}, h \in D\right\} .
$$

Put

$$
E(h):=\left(c_{h}, h\right) \in \mathcal{G}(\mathcal{K}, \psi)
$$

From (1.2) we get

$$
E(g) E(h)=\frac{c_{g} c_{h}}{c_{g+h}} \psi(g, h) E(g+h) .
$$

Assume that

$$
\alpha(g, h):=\frac{c_{g} c_{h}}{c_{g+h}} \psi(g, h)
$$

is a skewsymmetric pairing. Then the map $e_{D, \alpha}(h) \mapsto E(h)$ is compatible with the relations (1.6), i.e. defines a cohomologous representation of the Heisenberg group. In particular any representation $U$ of $\mathcal{G}(\mathcal{K}, \psi)$ induces a representation of an appropriate function algebra of the quantum torus $T(H, \alpha)$. One easily sees that any $\alpha$ on $D$ can be induced from an appropriate lattice embedding of $D$; one can even take $\psi$ to be a skewsymmetric bicharacter so that $\alpha$ will coincide with the restriction of $\psi$.

The condition (4.1) in the definition of formal exponents may be considered as a projective representation of $D$ with respect to the 2-cocycle $\alpha$. There is a canoncial correspondence between these representations and involutive representations of the twisted group algebra $\ell_{1}(D, \alpha)$ of $D$. It is known as the method of integrated representations.

The twisted group algebra $\ell_{1}(D, \alpha)$ of $D$ consists of all absolutely summable sequences $\mathbf{a}=\left(a_{h}\right)_{h \in D}$ where the multiplication is defined as twisted convolution of a and $\mathbf{b}$ by

$$
\mathbf{a} \sharp \mathbf{b}(h)=\sum_{l \in D} a_{l} b_{h-l} \alpha(h-l, l),
$$

and involution $\mathbf{a}^{*}=\left(a_{h}^{*}\right)_{h \in D}$ of $\mathbf{a}$ is defined by

$$
a_{h}^{*}=\overline{\alpha(h, h) a_{-h}} .
$$

Consequentely $\ell_{1}(D, \alpha)$ becomes a Banach algebra with respect to the norm $\|\mathbf{a}\|_{1}$.

If we "integrate" the formal exponents $\left\{e_{h}: h \in H\right\}$, then we get an involutive representation of $\ell_{1}(D, \alpha)$ as follows:

$$
\mathbf{a}=\left(a_{h}\right)_{h \in D} \mapsto \rho_{D}(\mathbf{a}):=\sum_{h \in D} a_{h} e_{D, \alpha}(h) .
$$

More explicitely, we have for all $\mathbf{a}, \mathbf{b}$ in $\ell_{1}(D)$ :

$$
\rho_{D}(\mathbf{a}) \rho_{D}(\mathbf{b})=\rho_{D}(\mathbf{a} \measuredangle \mathbf{b}) \text { and } \rho_{D}(\mathbf{a})^{*}=\rho_{D}\left(\mathbf{a}^{*}\right) \text {. }
$$


It is a well-known fact that there is a one-to-one correspondence between projective representations of $D$ and involutive representations of the twisted group algebra $\ell_{1}(D, \alpha)$. Finally we consider the twisted group $C^{*}$-algebra $C^{*}(D, \alpha)$ of $D$, which is the enveloping $C^{*}$-algebra of $\ell_{1}(D, \alpha)$.

Later we want to construct projective modules over smooth subalgebras $\mathcal{A}$ of $C^{*}(D, \alpha)$ in the sense of $\mathrm{A}$. Connes,. This means that $\mathcal{A}$ is stable under the holomorphic function calculus of $C^{*}(D, \alpha)$. The algebra $C^{\infty}(D, \alpha)$ of elements $\sum_{h \in D} a_{h} e_{D, \alpha}(h)$ with coefficients $\left\{a_{h}\right\}_{h \in D}$ belonging to the Schwartz space $\mathcal{S}(D)$ is a well-known example of a smooth subalgebra of $C^{*}(D, \alpha)$.

We want to point out that investigations in signal analysis have given rise to a whole class of smooth subalgebras of $C^{*}(D, \alpha)$, cf. [27]. They are denoted $C_{1}^{S}(D, \alpha)$, where one imposes on the elements $\sum_{h \in D} a_{h} e_{D, \alpha}(h)$ of $C^{*}(D, \alpha)$ the following summability conditions:

$$
\sum_{h \in D}\left|a_{h}\right|\left(1+|h|^{2}\right)^{s / 2}<\infty
$$

The fact that $C_{1}^{s}(D, \alpha)$ are smooth subalgebras of $C^{*}(D, \alpha)$ was shown by Gröchenig and Leinert in [27], where they proved the so-called irrationality conjecture of Gabor analysis. Recently J. Rosenberg has given another proof for the case $s=0$ of the theorem of Gröchenig and Leinert in [58].

Notice that $C^{\infty}(D, \alpha)=\bigcap_{s \geq 0} C_{1}^{s}(D, \alpha)$. In other words, one might consider $C_{1}^{S}(D, \alpha)$ as noncommutative analogues of differentable functions of order $s$. In [38], we have explored this point of view in detail.

Alternatively, any element of $C^{*}(D, \alpha)$ can also be written as a formal series $\sum_{h \in D} a_{h} e_{D, \alpha}(h)$ but there is no transparent way to specify which sequences $\left\{a_{h} \in\right.$ $\mathbf{C} \mid h \in D\}$ can occur as their "noncommutative Fourier coefficients". In this picture $C_{1}(D, \alpha)$ are noncommutative analogs of Wiener's algebra of Fourier series with absolutely convergent Fourier series. Recently Rosenberg wrote a very interesting paper [58] stressing this point of view. The theory of Gabor frames sheds some light on the structure of the noncommutative Fourier coefficients. It shows in particular that the class of modulation spaces has a natural characterization in terms of such noncommutative Fourier coeffiecients. We come back to this issue in a later section.

\subsection{PROJECTIVE MODULES OVER SMOOTH SUBALGEBRAS OF QUANTUM TORI}

In this section we discuss Rieffel's projective modules over $C^{\infty}(D, \alpha)$ and their extension to the setting of noncommutative Wiener algebras $C_{1}^{s}(D, \alpha)$, where $D$ is an embedded lattice of $\mathcal{K}$. Our presentation is inspired by the results in $[37,39,40$, 46,53-57].

In [53-56], it is shown that the space of smooth vectors $\mathcal{H}_{\infty}$ gives rise to a finitely generated projective $C^{\infty}(D, \alpha)$-module. Now we want to formulate the results of $[39,40]$ in this general framework. Therefore we introduce a family of 
subspaces of admissible elements $\mathcal{A}_{1}^{s}(\mathcal{K})$ for the Heisenberg group $\mathcal{G}(\mathcal{K}, \psi)$, as those elements $\mathbf{g}$ of $L_{2}(\mathcal{K})$ with $V_{\mathbf{g}}$ g in $L_{1}^{s}(\mathcal{K})$. Note that $\mathcal{H}_{\infty}=\bigcap_{s \geq 0} \mathcal{A}_{1}^{s}(\mathcal{K})$.

For $\Phi, \Psi$ in $\mathcal{A}_{1}^{s}(\mathcal{K})$

$$
{ }_{D}\langle\Phi, \Psi\rangle:=\sum_{h \in D}\left\langle\Phi, e_{D, \alpha}(h) \Psi\right\rangle e_{D, \alpha}(h)
$$

defines a scalar product with values in $C_{1}^{s}(D, \alpha)$, which is bounded on $\mathcal{A}_{1}^{s}(\mathcal{K})$. The space $\mathcal{A}_{1}^{s}(\mathcal{K})$ becomes a left pre-inner product $C_{1}^{s}(D, \alpha)$-module with respect to the following left action:

$$
\mathbf{a} \cdot \mathbf{f}=\sum_{h \in D} a_{h} e_{D, \alpha}(h) \cdot \mathbf{f},
$$

for $\mathbf{a} \in \ell_{1}^{s}(D)$ and $\mathbf{f} \in \mathcal{A}_{1}^{s}(\mathcal{K})$.

More explicitely, this means the following:

(i) Symmetry: ${ }_{D}\langle\Phi, \Psi\rangle^{*}={ }_{D}\langle\Psi, \Phi\rangle$.

(ii) (Bi)linearity: ${ }_{D}\langle a \Phi, \Psi\rangle=a_{D}\langle\Phi, \Psi\rangle$ for any $a \in C^{\infty}(D, \alpha)$.

(iii) Positivity: ${ }_{D}\langle\Phi, \Phi\rangle$ belongs to the cone of positive elements of $C^{*}(D, \alpha)$. Moreover, if ${ }_{D}\langle\Phi, \Phi\rangle=0$ then $\Phi=0$.

(iv) Density: The image of ${ }_{D}\langle$,$\rangle is dense in C_{1}^{S}(D, \alpha)$.

Consequently the completion of $\mathcal{A}_{1}^{s}(\mathcal{K})$ with respect to $\|\Phi\|:=\left\|{ }_{D}\langle\Phi, \Phi\rangle\right\|^{1 / 2}$ becomes a finitely generated left $C^{*}(D, \alpha)$-module $P$. Since $C_{1}^{s}(D, \alpha)$ is a smooth subalgebra of $C^{*}(D, \alpha)$ the finitely generated left $C_{1}^{S}(D, \alpha)$-module $P_{1}^{s}$ is isomorphic to $P$, see Lemma 4 on p. 52 of [6] and the discussion around Proposition 3.7 in [53-56].

As indicated above, this implies that $\mathcal{H}_{\infty}$ is also a projective finitely generated left $C^{\infty}(D, \alpha)$-module.

\subsection{PROJECTIVE MODULES OVER QUANTUM TORI FOR DUAL EMBEDDED LATTICES}

Let $D \subset \mathcal{K}$ be an embedded lattice as in 4.2. Denote by $D^{!}$the maximal closed subgroup of $\mathcal{K}$ orthogonal to $D$ with respect to $\varepsilon$. From the Pontryagin duality it follows that $D^{!}$(resp. $D$ ) can be canonically identified with the character group of $\mathcal{K} / D$ (resp. $\left.\mathcal{K} / D^{!}\right)$so that $D^{!}$is an embedded lattice as well.

Assume moreover that $\psi$ is an antisymmetric pairing, so that one can choose $E(h)=(1, h) \in \mathcal{G}(\mathcal{K}, \psi)$ for $h \in D$ and for $h \in D^{!}$and define on $\mathcal{A}_{1}^{s}(\mathcal{K})$ the structure of $C_{1}^{s}\left(D^{!}, \alpha^{!}\right)$-module as well where $\alpha^{!}$is the pairing induced on $D^{!}$by $\psi$. Operators $e_{D, \alpha}(h), h \in D$, commute with operators $e_{D^{!}, \alpha^{!}}(g), g \in D^{!}$.

We can consider $\mathcal{A}_{1}^{s}(\mathcal{K})$ as a right $C_{1}^{s}\left(D^{!}, \alpha^{!}\right)^{o p}$-module. Moreover, we can and will identify the latter algebra with $C_{1}^{s}\left(D^{!}, \bar{\alpha}^{!}\right)$by $e_{D^{!}, \alpha^{!}}(h) \mapsto e_{D^{!}, \bar{\alpha}^{!}}(h)^{-1}$ and extending this map by linearity. 
THEOREM 4.5. (i) We have $\left\|_{D}\langle\Phi, \Phi\rangle\right\|^{1 / 2}=\left\|_{D^{!}}\langle\Phi, \Phi\rangle\right\|^{1 / 2}$. The completion $\mathcal{H}$ of $\mathcal{A}_{1}^{s}(\mathcal{K})$ with respect to this norm is a projective left module over both quantum tori $C^{*}(D, \alpha)$ and $C^{*}\left(D^{!}, \alpha^{!}\right)$, and each of these algebras is a total commutator of the other one.

(ii) Let $C^{*}\left(D^{!}, \bar{\alpha}^{!}\right)$act upon $\mathcal{H}$ on the right as explained above. Consider the analog of the scalar product (4.13)

$$
\langle\Phi, \Psi\rangle_{D^{!}}:=\frac{1}{\text { volK } / D} \sum_{h \in D}\left\langle e_{D^{!}, \alpha^{!}}(h) \Psi, \Phi\right\rangle e_{D^{!}, \bar{\alpha}^{!}}(h) \in C^{*}\left(D^{!}, \bar{\alpha}^{!}\right)
$$

It satisfies relations similar to (i)-(iv), and moreover, for any $\Phi, \Psi, \Xi$ the following associativity relation holds:

$$
{ }_{D}\langle\Phi, \Psi\rangle \Xi=\Phi\langle\Psi, \Xi\rangle_{D^{!}}
$$

Consequently this result holds also for the smooth vectors $\mathcal{H}_{\infty}$ and the smooth subalgebra $C^{\infty}(D, \alpha)$. We discuss this theorem in the framework of Gabor analysis in a later section.

\section{Quantum Tori for Embedded Lattices and Gabor Analysis}

In the first part of this section we introduce the basic notions of Gabor analysis and in the second part we use this framework to interpret projective modules over quantum tori in terms of Gabor frames.

\subsection{BASICS OF GABOR ANALYSIS}

Recall that the square-integrability of the Schrödinger representation of the timefrequency plane yields the existence of a reconstruction formula for each $f \in$ $L_{2}\left(\mathbf{R}^{N}\right)$ :

$$
\mathbf{f}=\langle\mathbf{h}, \mathbf{g}\rangle^{-1} \iint_{\mathbf{R}^{2 N}} V_{\mathbf{g}} \mathbf{f}(x, \omega) \pi(x, \omega) \mathbf{h} \mathrm{d} x \mathrm{~d} \omega,
$$

for $\mathbf{g}, \mathbf{h} \in L_{2}\left(\mathbf{R}^{N}\right)$ with $\langle\mathbf{g}, \mathbf{h}\rangle \neq 0$.

In the reconstruction formula (5.1) the time-frequency content of a signal $\mathbf{f}$ is analysed with respect to the system $\left\{\pi(x, \omega) \mathbf{g}:(x, \omega) \in \mathbf{R}^{N}\right\}$ for a window $\mathbf{g} \in$ $L_{2}\left(\mathbf{R}^{N}\right)$, i.e. one considers the STFT $\left(\langle\mathbf{f}, \pi(x, \omega) \mathbf{g}\rangle:(x, \omega) \in \mathbf{R}^{N}\right)$ for each building block $\pi(x, \omega) \mathbf{g}$, and then this information about the signal is used in the synthesis process with respect to the system $\left\{\pi(x, \omega) \mathbf{h}:(x, \omega) \in \mathbf{R}^{N}\right\}$. We call the function $\mathbf{h}$ the synthesis window. Note that there is just one restriction on the synthesis window, namely $\mathbf{h}$ has to be non-orthogonal to the window $\mathbf{g}$. The reconstruction formula (5.1) is well-known in quantum mechanics, where it is refered to as resolution of identity. 
In 1946, D. Gabor was looking for an "optimal" way to transmit a signal [23]. Therefore he wanted to maximize the content of information gained from the analysis process and to minimize the synthesis process. First, he suggested to use well-localized window functions. Since the Gaussian $\mathbf{g}_{0}(t)=\mathrm{e}^{-\pi t^{2}}$ (and its timefrequency shifts) is the minimizer of the Heisenberg uncertainty principle, Gabor relied his investigations on the Gaussian as window function. Second, Gabor considered discrete analogues of the resolution of identity, where he replaced the time-frequency plane $\mathbf{R}^{2 N}$ by the lattice $\mathbf{Z}^{2 N}$. Relying upon an heuristic argument Gabor claimed that each $\mathbf{f} \in L_{2}\left(\mathbf{R}^{N}\right)$ has an expansion of the following type

$$
\mathbf{f}=\sum_{k, m \in \mathbf{Z}^{N}}\left\langle\mathbf{f}, \pi(k, m) \mathbf{g}_{0}\right\rangle \pi(k, m) \mathbf{h},
$$

for some $\mathbf{h}$ in $L_{2}\left(\mathbf{R}^{N}\right)$.

Until the late 1970s Gabor's paper [23] drew little attention of engineers and mathematicians, because the actual implementation of Gabor's expansions did not perform very well. In a series of papers [31-33] a mathematician Janssen undertook a rigorous investigation of Gabor's original expansions (5.2). The main result in [31] shows that the series (5.2) converges for $\mathbf{f}$ and $\mathbf{g}$ not in $L_{2}\left(\mathbf{R}^{N}\right)$, because the coefficients turn out to grow logarithmically. Janssen proved instead convergence in the sense of tempered distributions and thereby explained why the expansions (5.2) are numerically unstable. The main reason for the problems with Gabor's original proposal is that the corresponding system does not give a frame for $L^{2}\left(\mathbf{R}^{N}\right)$.

After the rigorous analysis of (5.2) mathematicians and engineers started looking for systems of functions in $L_{2}\left(\mathbf{R}^{N}\right)$ that would allow one to get numerically stable expansions of Gabor's type. The great breakthrough in this direction was the work [8] in 1986, where the authors demonstrated that so-called frames of a Hilbert space provide stable reconstruction formulas.

The notion of frames of a Hilbert space had been already introduced by Duffin and Schaeffer in their work on non-harmonic Fourier series [11]. Let $\mathcal{H}$ be a separable Hilbert space. Then a system $\left\{\mathbf{g}_{i} \mid i \in I\right\}$ is a frame for $\mathcal{H}$ if and only if for each $\mathbf{f} \in \mathcal{H}$ the following holds: there exist finite positive constants $A, B$ such that

$$
A\|\mathbf{f}\|_{\mathcal{H}}^{2} \leq \sum_{i \in I}\left|\left\langle\mathbf{f}, \mathbf{g}_{i}\right\rangle\right|^{2} \leq B\|\mathbf{f}\|_{\mathcal{H}}^{2} .
$$

In $[8,9]$ the authors investigated two kinds of frames generated by (i) Gabor systems and (ii) wavelets. Both systems are so-called atomic decomposition, because the elements of the systems are generated out of a building block $\mathbf{g}$ by the action of a group representation. A relevant group is the Heisenberg group in the case of Gabor frames and the affine group for wavelet systems. Shortly after the groundbreaking work of Daubechies, Grossman and Meyer, the mathematicians Feichtinger and Gröchenig realized that Gabor systems and wavelets are just two examples of a general framework, which culminated in their coorbit theory [17] and revealed the great relevance of representation theory for the construction of frames. 
After this brief historical review we continue our discussion of Gabor frames. Since the work of Feichtinger and Gröchenig, a Gabor system $\mathbf{G}(\mathbf{g}, D)$ is defined as the set of time-frequency shifts of a given Gabor atom $\mathbf{g}$ for an embedded lattice $D$ in $\mathbf{R}^{2 N}$, i.e.

$$
\mathbf{G}(\mathbf{g}, D)=\{\pi(h) \mathbf{g}: h \in D\} .
$$

A Gabor system $\mathbf{G}(\mathbf{g}, D)$ is called a Gabor frame for $L_{2}\left(\mathbf{R}^{N}\right)$ if satisfies (5.3) for some constants $A, B$.

The main task of Gabor analysis is to find reconstruction formulas for a function $\mathbf{f}$ in terms of $\mathbf{G}(\mathbf{g}, D)$.

The following operators associated to a Gabor system $\mathbf{G}(g, \Lambda)$ allow one to write down such reconstruction formulas.

(i) The analysis operator $C_{\mathbf{g}, D}$ maps functions $\mathbf{f} \in L_{2}\left(\mathbf{R}^{N}\right)$ to sequences on $D$ by

$$
C_{\mathbf{g}, D} \mathbf{f}(h)=\langle\mathbf{f}, \pi(h) \mathbf{g}\rangle_{h \in D} .
$$

(ii) The synthesis operator maps sequences $\mathbf{c}=\left(c_{h}\right)$ on $D$ to functions on $\mathbf{R}^{N}$ as follows:

$$
\mathbf{c} \mapsto \sum_{h \in D} c_{h} \pi(h) \mathbf{g} .
$$

The Gabor frame operator $S_{\mathbf{g}, D}$ corresponding to the Gabor system $\mathbf{G}(\mathbf{g}, D)$ maps a function $\mathbf{f}$ to

$$
S_{\mathbf{g}, D} \mathbf{f}=\sum_{h \in D}\langle\mathbf{f}, \pi(h) \mathbf{g}\rangle \pi(h) \mathbf{g} .
$$

Observe that $S_{\mathbf{g}, D}$ is the composition of the analysis operator followed by the synthesis operator. Observe that $\mathbf{G}(\mathbf{g}, D)$ is a Gabor frame for $L_{2}\left(\mathbf{R}^{N}\right)$ if and only if the Gabor frame operator $S_{\mathbf{g}, D}$ is invertible on $L_{2}\left(\mathbf{R}^{N}\right)$.

Now, the existence of reconstruction formulas for $\mathbf{f}$ is linked to the invertibility of the Gabor frame operator $S_{\mathrm{g}, D}$. We define the canonical dual Gabor atom and the canonical tight Gabor atom of the Gabor frame $\mathbf{G}(\mathbf{g}, D)$ by $\tilde{\mathbf{g}}:=S_{\mathbf{g}, D}^{-1} \mathbf{g}$ and $\tilde{\mathbf{h}}=S_{\mathbf{g}, D}^{-1 / 2} \mathbf{g}$, respectively. Then we have the following reconstruction formulas for $\mathbf{f} \in$ $L_{2}\left(\mathbf{R}^{N}\right)$ :

$$
\mathbf{f}=\sum_{h \in D}\langle\mathbf{f}, \pi(h) \mathbf{g}\rangle \pi(h) \tilde{\mathbf{g}}
$$

and

$$
\mathbf{f}=\sum_{h \in D}\langle\mathbf{f}, \pi(h) \tilde{\mathbf{h}}\rangle \pi(h) \tilde{\mathbf{h}} .
$$


For $\mathbf{g}, \mathbf{h} \in L_{2}\left(\mathbf{R}^{N}\right)$ we call

$$
S_{\mathbf{g}, \mathbf{h}, D} \mathbf{f}=\sum_{h \in D}\langle\mathbf{f}, \pi(h) \mathbf{h}\rangle \pi(h) \mathbf{g}
$$

a Gabor frame-type operator. These operators appear naturally in reconstruction formulas, e.g. if $\tilde{\mathbf{g}}=S_{\mathbf{g}, D}^{-1} \mathbf{g}$ then $S_{\mathbf{g}, \tilde{\mathbf{g}}, D}=I$.

Around 1995 the papers [10,34,59] developed the so-called duality theory of Gabor analysis, which marked a turning point in Gabor analysis. At the heart of all these contributions is the crucial observation, that a Gabor frame operator has a decomposition with respect to another Gabor system, the so-called Janssen representation of the Gabor frame operator. This relies on the observation that the Gabor frame operator $S_{\mathbf{g}, D}$ commutes with $\pi(h)$ for $h \in D$ :

$$
\pi(h) S_{\mathbf{g}, D}=S_{\mathbf{g}, D} \pi(h) \quad h \in D .
$$

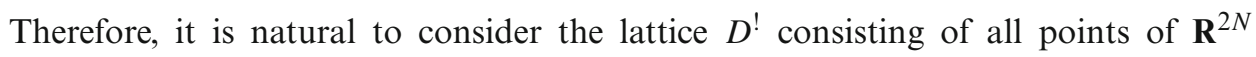
commuting with $\{\pi(h): h \in D\}$ :

$$
D^{!}=\left\{h^{!} \in \mathbf{R}^{2 N}: \pi(h) \pi\left(h^{!}\right)=\pi\left(h^{!}\right) \pi(h) \text { for all } h \in D\right\} .
$$

The lattice $D^{!}$is the so-called adjoint lattice, which had been introduced by Feichtinger and Kozek [19] in their discussion of the Janssen representation. In Gabor analysis the adjoint lattice is usually denoted by $D^{\circ}$. In other contexts this object has appeared in the work of Mumford on theta functions and polarized abelian varieties, Rieffel's construction of projective modules over noncommutative tori [53-56]. Note that the dual embedded lattice in the previous section is the adjoint lattice for the Heisenberg group $\mathbf{G}\left(\mathbf{R}^{2 N}, \psi_{I}\right)$.

Consider $\mathbf{g}, \mathbf{h} \in L_{2}\left(\mathbf{R}^{N}\right)$ satisfying the following condition of Tolimieri and Orr:

$$
\sum_{h^{!} \in D^{!}}\left|\left\langle\mathbf{g}, \pi\left(h^{!}\right) \mathbf{h}\right\rangle\right|<\infty .
$$

Then the Gabor frame-type operator $S_{g, h, D}$ has the following Janssen representation:

$$
S_{\mathbf{g}, \mathbf{h}, D} f=\operatorname{vol}(D)^{-1} \sum_{h^{!} \in D^{!}}\left\langle\mathbf{h}, \pi\left(h^{!}\right) \mathbf{g}\right\rangle \pi\left(h^{!}\right) \mathbf{f},
$$

where $\operatorname{vol}(D)$ denotes the volume of a fundamental domain of $D$.

The Janssen representation follows from the Fundamental Identity of Gabor analysis (FIGA) after an application of a symplectic Poisson summation formula, see (5.18) below. The validity of Poisson summation formulas is a delicate matter. In the present situation the space of admissible vectors of the vector Heisenberg group $\mathcal{G}\left(\mathbf{R}^{2 N}, \psi_{A}\right)$ provides a good class of functions. Due to its great importance in harmonic analysis, time-frequency analysis and approximation theory this space [14] is known as Feichtinger's algebra $S_{0}\left(\mathbf{R}^{N}\right)$. We recall the definition of the 
weighted variants of Feichtinger's algebra $M_{1}^{S}\left(\mathbf{R}^{N}\right)$, these are elements of the class of modulation spaces [14]. The tempered distribution $\mathbf{f} \in \mathcal{S}^{\prime}\left(\mathbf{R}^{N}\right)$ is in $M_{1}^{S}\left(\mathbf{R}^{N}\right)$ if

$$
\|\mathbf{f}\|_{M_{1}^{s}}:=\iint_{\mathbf{R}^{2 N}}\left|V_{\mathbf{g}} \mathbf{f}(x, \omega)\right|\left(1+\left|x^{2}\right|\right)^{s / 2} \mathrm{~d} x \mathrm{~d} \omega<\infty
$$

for a window $\mathbf{g}$ in $\mathcal{S}\left(\mathbf{R}^{N}\right)$. The defintion of $M_{1}^{S}\left(\mathbf{R}^{N}\right)$ is independent of the window function $\mathbf{g}$. It is a Banach space invariant under time-frequency shifts. The space $M_{1}\left(\mathbf{R}^{N}\right)$ is Feichtinger's algebra $S_{0}\left(\mathbf{R}^{N}\right)$, which is the minimal element in the class of Banach spaces invariant under time-frequency shifts.

We formulate the symplectic Poisson summation formula in the following statement:

PROPOSITION 5.1.1. Let $D$ be an embedded lattice and $D^{!}$its adjoint lattice in $\mathbf{R}^{2 N}$. Then for $\mathbf{F}$ in $M_{1}\left(\mathbf{R}^{2 N}\right)$ or $\mathbf{F}$ in $\mathcal{S}\left(\mathbf{R}^{N}\right)$ we have

$$
\sum_{h \in D} \mathbf{F}(h)=\frac{1}{\operatorname{vol}(D)} \sum_{h ! \in D^{!}} \mathbf{F}\left(h^{!}\right) .
$$

This holds pointwise, and the convegence is absolute.

Therefore we are able to obtain the FIGA, which is a consequence of the symplectic Poisson summation formula for $\mathbf{F}=V_{\mathbf{g}_{1}} \mathbf{f}_{1} \cdot V_{\mathbf{g}_{2}} \mathbf{f}_{2}$. We just have to find conditions on the windows and functions guaranteeing that the product of the two STFT's is in $M_{1}\left(\mathbf{R}^{N}\right)$ or in $\mathcal{S}\left(\mathbf{R}^{N}\right)$.

PROPOSITION 5.1.2. Let $\mathbf{f}_{1}, \mathbf{f}_{2}, \mathbf{g}_{1}, \mathbf{g}_{2}$ be in $M_{1}\left(\mathbf{R}^{N}\right)$ or in $\mathcal{S}\left(\mathbf{R}^{N}\right)$. Then we have

$$
\sum_{h \in D}\left(V_{\mathbf{g}_{1}} \mathbf{f}_{1} V_{\mathbf{g}_{2}} \mathbf{f}_{2}\right)(h)=\frac{1}{\operatorname{vol}(D)} \sum_{h ! \in D^{!}}\left(V_{\mathbf{g}_{1}} \mathbf{g}_{2} V_{\mathbf{f}_{1}} \mathbf{f}_{2}\right)\left(h^{!}\right) .
$$

The Janssen representation of $S_{\mathrm{g}, D}$ is a direct consequence of (5.18). We consider $\left\langle S_{\mathbf{g}, D} \mathbf{f}, \mathbf{k}\right\rangle$ and observe that this is the left hand side of (5.18). Therefore (5.18) yields that

$$
\left\langle S_{\mathbf{g}, D} \mathbf{f}, \mathbf{k}\right\rangle=\left\langle\frac{1}{\operatorname{vol}(D)} \sum_{h^{!} \in D^{!}}\left(V_{\mathbf{g}} \mathbf{g} \cdot V_{\mathbf{f}} \mathbf{k}\right)\left(h^{!}\right)\right\rangle .
$$

and we can write the right hand side of (5.19) in the following way:

$$
\sum_{d^{!} \in D^{!}}\left(V_{\mathbf{g}} \mathbf{g} \cdot V_{\mathbf{f}} \mathbf{h}\right)\left(d^{!}\right)=\left\langle\sum_{d^{!} \in D^{!}}\left\langle\mathbf{g}, \pi\left(d^{!}\right) \mathbf{g}\right\rangle \pi\left(d^{!}\right) \mathbf{f}, \mathbf{h}\right\rangle .
$$

From this we derive the Janssen representation of $S_{\mathrm{g}, D}$ : 
PROPOSITION 5.1.2. Let $\mathbf{f}, \mathbf{g}, \mathbf{k}$ be in $M_{1}\left(\mathbf{R}^{N}\right)$ or in $\mathcal{S}\left(\mathbf{R}^{N}\right)$. Then

$$
S_{\mathbf{g}, D} \mathbf{f}=\frac{1}{\operatorname{vol}(D)} \sum_{d^{!} \in D^{!}}\left\langle\mathbf{g}, \pi\left(d^{!}\right) \mathbf{g}\right\rangle \pi\left(d^{!}\right) \mathbf{f} .
$$

The Janssen representation lies at the heart of the duality theory for Gabor frames, which was developed independently by three groups of researchers $[10$, $34,59]$ at the same time. Later Feichtinger and Gröchenig extended their $L_{2}(\mathbf{R})$ results. These results are the cornerstones of Gabor analysis. One important consequence of (5.21) is that the invertibility of $S_{\mathbf{g}, D}$ becomes a question about the invertibility of an absolutely convergent sequence of time-frequency shifts:

$$
S_{\mathbf{g}, D}=\frac{1}{\operatorname{vol}(D)} \sum_{h^{!} \in D^{!}}\left\langle\mathbf{g}, \pi\left(h^{!}\right) \mathbf{g}\right\rangle \pi\left(h^{!}\right) .
$$

In [27] Gröchenig and Leinert were able to link this fact with the spectral invariance of subalgebras of the quantum torus $C^{*}\left(D^{!}, \alpha\right)$. Later we observed that these kind of results are of great relevance for noncommutative geometry, especially some theorems of Connes on smooth quantum tori [38].

Observe that all our statements involving $M_{1}\left(\mathbf{R}^{N}\right)$ in this section also hold for $M_{1}^{s}\left(\mathbf{R}^{N}\right)$. Therefore by the time-frequency description of the smooth vectors of the vector Heisenberg group, i.e. the Schwartz class $\mathcal{S}\left(\mathbf{R}^{N}\right)=\bigcap_{s \geq 0} M_{1}^{s}\left(\mathbf{R}^{N}\right)$, the spaces $\left\{M_{1}^{S}\left(\mathbf{R}^{N}\right) \mid s \geq 0\right\}$ provide a scale of Banach spaces that interpolates between the integrable vectors and the smooth vectors.

\subsection{PROJECTIVE MODULES OVER QUANTUM TORI IN THE SETTING OF GABOR} ANALYSIS

In our treatment of the Janssen representation we emphasized the relevance of FIGA and that it already appeared in Rieffel's construction of projective modules over noncommutative tori. Now we want to exploit this link between projective modules over quantum tori and Gabor frames in more detail, see [37,39,40] for further aspects of this topic.

The link between abstract quantum tori from Section 4 and Gabor analysis is provided by the choice of a particular representation for the quantum torus. Namely the operators $\{\pi(d): d \in D\}$ extend to a faithful involutive representation of the quantum torus $C^{*}(D, \alpha)$ on $L_{2}\left(\mathbf{R}^{N}\right)$. Consequently the smooth subalgebras $C_{1}^{S}(D, \alpha)$ and $C^{\infty}(D, \alpha)$ become the following classes of operator algebras:

$$
C_{1}^{s}(D, \alpha)=\left\{\sum_{h \in D} a_{h} \pi(h)\left|\sum_{h \in D}\right| a_{h} \mid\left(1+|h|^{2}\right)^{s / 2}<\infty\right\}
$$

and $C^{\infty}(D, \alpha)=\bigcap_{s \geq 0} C_{1}^{s}(D, \alpha)$. By the integrated Heisenberg commutation relations the 2-cocycle $\alpha$ in this representation take the following form: $\alpha(h, k)=$ $\mathrm{e}^{2 \pi i h_{\omega} \cdot k_{x}}$ for $h=\left(h_{x}, h_{\omega}\right)$ and $k=\left(k_{x}, k_{\omega}\right)$. 
In other words $C_{1}^{s}(D, \alpha)$ consists of the image of the integrated representation of $\ell_{1}^{s}(D)$ with respect to the faithful involutive representation generated by the time-frequency shifts $\pi(h)$. We denote the integrated representation of $\mathbf{a} \in \ell_{1}^{s}(D)$ by $\pi_{D}(\mathbf{a})$ :

$$
\pi_{D}(\mathbf{a})=\sum_{h \in D} a_{h} \pi(h) .
$$

In this setting the left action of $C_{1}^{S}(D, \alpha)$ on $\mathbf{g} \in M_{1}^{s}\left(\mathbf{R}^{N}\right)$ becomes

$$
\mathbf{a} \cdot \mathbf{g}=\pi_{D}(\mathbf{a}) \cdot \mathbf{g}=\sum_{h \in D} a_{h} \pi(h) \mathbf{g},
$$

for $\mathbf{a} \in \ell_{1}^{s}(D, \alpha)$ and similarly for $C^{\infty}(D, \alpha)$ acting on the left on $\mathcal{S}\left(\mathbf{R}^{N}\right)$ for $\mathbf{a} \in$ $\mathcal{S}(D, \alpha)$.

The algebra-valued product on $C_{1}^{s}(D, \alpha)$ turns out to be

$$
{ }_{D}\langle\mathbf{f}, \mathbf{g}\rangle=\sum_{h \in D}\langle\mathbf{f}, \pi(h) \mathbf{g}\rangle \pi(h),
$$

for $\mathbf{f}, \mathbf{g} \in M_{1}^{S}\left(\mathbf{R}^{N}\right)$ and similarly on $C^{\infty}(D, \alpha)$ for $\mathbf{f}, \mathbf{g} \in \mathcal{S}\left(\mathbf{R}^{N}\right)$.

Note that the left action (5.25) is just the synthesis operator of the Gabor system $\mathbf{G}(\mathbf{g}, D)$ for $\mathbf{g} \in M_{1}^{S}\left(\mathbf{R}^{N}\right)$ or in $\mathcal{S}\left(\mathbf{R}^{N}\right)$, and that the scalar product is the integrated representation of the sequence obtained from the analysis mapping of the Gabor system $\mathbf{G}(\mathbf{g}, D)$.

PROPOSITION 5.1.3. Let $D$ be an embedded lattice of $\mathbf{R}^{2 N}$. Then $M_{1}^{S}\left(\mathbf{R}^{N}\right)$ and $\mathcal{S}\left(\mathbf{R}^{N}\right)$ become a left Hilbert $C^{*}(D, \alpha)$-module ${ }_{D} V$ after completing by the norm ${ }_{D}\|\mathbf{f}\|=\left\|_{D}\langle\mathbf{f}, \mathbf{f}\rangle\right\|^{1 / 2}$.

We refer the reader to $[39,40,53-56]$ for a proof and generalizations of the last proposition. The most important operators on Hilbert $C^{*}$-modules are the rankone Hilbert module operators, which in our case are defined by

$$
\Theta_{\mathbf{g}, \mathbf{k}}^{D} \mathbf{f}:={ }_{D}\langle\mathbf{f}, \mathbf{g}\rangle \cdot \mathbf{k}=\sum_{h \in D}\langle\mathbf{f}, \pi(h) \mathbf{g}\rangle \pi(h) \mathbf{k} .
$$

Operators of the form $\Theta_{\mathbf{g}, \mathbf{k}}^{D} \mathbf{f}$ are called Gabor frame-type operators for a given Gabor system $\mathbf{G}(\mathbf{g}, D)$ and are denoted by $S_{\mathbf{g}, \mathbf{k}, D}$. These operators appear naturally in the discrete reconstruction formulas.

In the following we want to discuss the Rieffel-Morita equivalence of $C^{*}(D, \alpha)$ and $C^{*}\left(D^{!}, \bar{\alpha}^{\prime}\right)$. Recall that two $C^{*}$-algebras $\mathcal{A}$ and $\mathcal{B}$ are called Rieffel-Morita equivalent, if there exists an $\mathcal{A}$ - $\mathcal{B}$-equivalence bimodule $\mathcal{A}_{\mathcal{A}} V_{\mathcal{B}}$ such that:

(i) ${ }_{\mathcal{A}} V_{\mathcal{B}}$ is a full left Hilbert $\mathcal{A}$-module and a full right Hilbert $\mathcal{B}$-module;

(ii) for all $f, g \in \mathcal{A} V_{\mathcal{B}}, A \in \mathcal{A}$ and $B \in \mathcal{B}$ we have that

$$
\langle A \cdot f, g\rangle_{\mathcal{B}}=\left\langle f, A^{*} \cdot g\right\rangle_{\mathcal{B}} \text { and } \mathcal{A}\langle f \cdot B, g\rangle=\mathcal{A}\left\langle f, g \cdot B^{*}\right\rangle ;
$$


(iii) for all $f, g, h \in_{\mathcal{A}} V_{\mathcal{B}}$,

$$
\mathcal{A}\langle f, g\rangle \cdot h=f \cdot\langle g, h\rangle_{\mathcal{B}} .
$$

We refer the reader to [49] for an extensive discussion of Rieffel-Morita equivalence.

We continue with a discussion of the right module structure on $M_{1}^{s}\left(\mathbf{R}^{N}\right)$ or $\mathcal{S}\left(\mathbf{R}^{N}\right)$. Since the quantum torus is only Morita equivalent to the opposite algebra torus $C^{*}\left(D^{!}, \bar{\alpha}^{!}\right)$, the action and the scalar product $\langle., .\rangle_{D^{!}}$differ from those in the case of $C^{*}(D, \alpha)$. More precisely, for $\mathbf{b} \in \ell_{1}^{s}\left(D^{!}\right), \mathbf{f}, \mathbf{g}$ in $M_{1}^{s}\left(\mathbf{R}^{N}\right)$ we define a right action of $C_{1}^{s}\left(D^{!}, \bar{\alpha}^{!}\right)$on $M_{1}^{s}\left(\mathbf{R}^{N}\right)$ by

$$
\mathbf{g} \cdot \mathbf{b}:=\mathbf{g} \cdot \pi_{D^{!}}(\mathbf{b})=\frac{1}{\operatorname{vol}(D)} \sum_{h^{\prime} \in D^{!}} \pi\left(h^{!}\right) \mathbf{f} \overline{b\left(h^{!}\right)},
$$

and the $C_{1}^{S}\left(D^{!}, \bar{\alpha}^{!}\right)$-valued scalar product by

$$
\langle\mathbf{f}, \mathbf{g}\rangle_{D^{!}}=\sum_{h^{!} \in D^{!}} \pi\left(h^{!}\right)^{*}\left\langle\pi\left(h^{!}\right) \mathbf{g}, \mathbf{f}\right\rangle .
$$

As indicated above this yields also a right action and a $C^{\infty}\left(D^{!}, \bar{\alpha}^{!}\right)$-valued scalar product in the case of $\mathcal{S}\left(\mathbf{R}^{N}\right)$. Consequentely, we get a right Hilbert $C^{*}\left(D^{!}, \bar{\alpha}^{!}\right)$module $V_{D^{!}}$after completing $M_{1}^{S}\left(\mathbf{R}^{N}\right)$ or $\mathcal{S}\left(\mathbf{R}^{N}\right)$ by $\|\mathbf{f}\|_{D^{!}}=\left\|\langle\mathbf{f}, \mathbf{f}\rangle_{D^{!}}\right\|^{1 / 2}$.

The main result of [53-56] asserts that these two Hilbert $C^{*}$-modules form actually an equivalence bimodule, and the two scalar products ${ }_{D} !\langle.,$.$\rangle and \langle., .\rangle_{D^{!}}$satisfy the associativity equation:

$$
{ }_{D}\langle\mathbf{f}, \mathbf{g}\rangle \cdot \mathbf{k}=\mathbf{f} \cdot\langle\mathbf{g}, \mathbf{k}\rangle_{D} !
$$

for $\mathbf{f}, \mathbf{g}, \mathbf{k}$ in $M_{1}^{S}\left(\mathbf{R}^{N}\right)$ or $\mathcal{S}\left(\mathbf{R}^{N}\right)$, respectively. Recall our discussion of the Janssen representation of $S_{\mathrm{g}, D}$. If you consider the Janssen representation of a Gabor frame-type operator $S_{\mathbf{g}, \mathbf{k}, D}$, then you get the associativity condition (5.30).

PROPOSITION 5.1.4. Let $D$ be an embedded lattice of $\mathbf{R}^{2 N} . M_{1}^{S}\left(\mathbf{R}^{N}\right)$ and $\mathcal{S}\left(\mathbf{R}^{N}\right)$ become an $C^{*}(D, \alpha)-C^{*}\left(D^{!}, \bar{\alpha}^{!}\right)$equivalence bimodule ${ }_{D} V_{D^{!}}$after completing by the norm ${ }_{D}\|\mathbf{f}\|=\left\|_{D}\langle\mathbf{f}, \mathbf{f}\rangle\right\|^{1 / 2}$.

The involutive Banach algebras $C_{1}^{s}(D, \alpha)$ and the smooth noncommutative torus $C^{\infty}(D, \alpha)$ are spectrally invariant subalgebras of the quantum torus $C^{*}(D, \alpha)$, i.e. if $A \in C_{1}^{S}(D, \alpha)$ or $C^{\infty}(D, \alpha)$ is invertible in $C^{*}(D, \alpha)$, then $A^{-1}$ is in $C_{1}^{s}(D, \alpha)$ or $C^{\infty}(D, \alpha)$, respectively. The spectral invariance of $C_{1}^{S}(D, \alpha)$ was recently proved by Gröchenig and Leinert in [27]. For $C^{\infty}(D, \alpha)$ this is a famous theorem of Connes [5].

In [6] it is demonstrated that if there exists a subspace $V_{0}$ of the equivalence bimodule ${ }_{D} V_{D^{!}}$, that is invariant under the left and right actions of $\mathcal{A}_{0}$ and $\mathcal{B}_{0}$, 
and such that the scalar products evaluated for elements in $V_{0}$ are elements of spectrally invariant subalgebras $\mathcal{A}_{0}$ and $\mathcal{B}_{0}$ of $C^{*}(D, \alpha)$ and $C^{*}\left(D^{!}, \bar{\alpha}^{!}\right)$, then $V_{0}$ is a projective left $\mathcal{A}_{0}$-module and projective right $\mathcal{B}_{0}$-module respectively. We call the two involutive algebras $\mathcal{A}_{0}$ and $\mathcal{B}_{0}$ Rieffel-Morita equivalent, if there exists such an equivalence bimodule $V_{0}$.

The spaces $M_{1}^{S}\left(\mathbf{R}^{N}\right)$ and the noncommutative Wiener algebras $C_{1}^{S}(D, \alpha)$ fulfill these requirements. Therefore we get

THEOREM 5.1.5. The noncommutative Wiener algebras $C_{1}^{s}(D, \alpha)$ and $C_{1}^{s}\left(D^{!}, \bar{\alpha}^{!}\right)$ are Rieffel-Morita equivalent through the modulation space $M_{1}^{s}\left(\mathbf{R}^{N}\right)$ for all $s \geq 0$. Consequently, the noncommutative smooth tori $C^{\infty}(D, \alpha)$ and $C^{\infty}\left(D^{!}, \bar{\alpha}^{!}\right)$are Rieffel-Morita equivalent through the Schwartz space $\mathcal{S}\left(\mathbf{R}^{N}\right)$.

In [40] we generalize these results to other classes of spectrally invariant subalgebras of $C^{*}(D, \alpha)$ with subexponential growth.

\section{Quantum Theta Functions and their Functional Equations}

For the treatment of quantum theta functions we have to introduce the class of generalized Gaussians, which appear prominently in various areas, e.g. in quantum optics as squeezed states, in harmonic analysis [21], the work of Littlejohn on semi-classical mechanics in [36], and in de Gosson's work on symplectic capacity as measure of uncertainty in quantum mechanics [24].

In the description of the $I I_{T}$-model in 2.2 above, we have implicitly used the Siegel upper-half plane $\mathcal{S}_{N}$ which is the space of all matrices of the form $T=\operatorname{Re} T+$ $i \operatorname{Im} T$, where $\operatorname{Re} T, \operatorname{ImT}$ are real symmetric $N \times N$-matrices, and $\operatorname{Im} T$ is positive definite. Then we define a generalized Gaussian by

$$
\mathbf{g}_{T}(x)=\mathrm{e}^{-\langle T x, x\rangle}=\mathrm{e}^{-\langle(\operatorname{Re} T+i \operatorname{Im} T) x, x\rangle} \quad \text { for } x \in \mathbf{R}^{N} .
$$

In his work on quantum theta functions Manin [43-45] has calculated the Wigner transform of generalized Gaussians, which is a well-known result in quantum optics and quantum mechanics.

Recall that the Wigner distribution $W(\mathbf{g}, \mathbf{g})$ of a function $g$ is given by

$$
W(\mathbf{g}, \mathbf{g})(x, \omega)=\int_{\mathbf{R}^{N}} \mathbf{g}\left(x+\frac{t}{2}\right) \overline{\mathbf{g}}\left(x-\frac{t}{2}\right) \mathrm{e}^{-2 \pi i t \cdot \omega} \mathrm{d} t
$$

and that the Wigner distribution is just a short-time Fourier transform in disguise:

$$
W(\mathbf{g}, \mathbf{g})(x, \omega)=2^{N} \mathrm{e}^{4 \pi i x \cdot \omega} V_{\tilde{\mathbf{g}}} \mathbf{g}(2 x, 2 \omega),
$$

where $\tilde{\mathbf{g}}(x)=\mathbf{g}(-x)$ denotes the reflection of $\mathbf{g}$ with respect to the origin, see (3.8) and (3.9). 
Probably, one of the earliest calculations of the Wigner transform of a generalized Gaussian has been published by the electrical engineer Bastiaans [1].

LEMMA 6.1. Let $\mathbf{g}_{T}$ be the generalized Gaussian for a $T \in \mathcal{S}_{N}$. Then the Wigner transform of $\mathbf{g}_{T}$ is given by

$$
W\left(\mathbf{g}_{T}, \mathbf{g}_{T}\right)(z)=(\operatorname{det} T)^{-1 / 2} \mathrm{e}^{-H_{T}(z, z)}
$$

where $H_{T}(z, z)=G_{T} z \cdot z$, and

$$
G_{T}=\left(\begin{array}{cc}
\operatorname{Re} T+\operatorname{Im} T(\operatorname{Re} T)^{-1} \operatorname{Im} T & \operatorname{Im} T(\operatorname{Re} T)^{-1} \\
(\operatorname{Re} T)^{-1} \operatorname{Im} T & (\operatorname{Re} T)^{-1}
\end{array}\right)
$$

The matrix $G_{T}$ is symplectic and has the following factorization: $G_{T}=S^{T} S$ with

$$
S=\left(\begin{array}{cc}
(\operatorname{Re} T)^{1 / 2} & 0 \\
(\operatorname{Re} T)^{-1 / 2} \operatorname{Im} T & (\operatorname{Re} T)^{-1 / 2}
\end{array}\right) .
$$

A generalized Gaussian $\mathbf{g}_{T}$ is an element of $\mathcal{S}\left(\mathbf{R}^{N}\right)$, because it belongs also to $M_{s}^{1}\left(\mathbf{R}^{N}\right)$ for any $s \geq 0$. The last observation follows from a well-known characterization of $M_{s}^{1}\left(\mathbf{R}^{N}\right)$ in terms of the Wigner transform. Namely, $\mathbf{g} \in M_{s}^{1}\left(\mathbf{R}^{N}\right)$ if and only if $W(\mathbf{g}, \mathbf{g})$ is in $L_{s}^{1}\left(\mathbf{R}^{N}\right)$. An elementary computation yields that the Wigner distribution of $\mathbf{g}_{T}$ is in $L_{s}^{1}\left(\mathbf{R}^{N}\right)$ for any $s \geq 0$. We combine this observation and its interesting consequence in the next lemma.

LEMMA 6.2. Let $D$ be an embedded sublattice of $\mathbf{R}^{2 N}$. Since $\mathbf{g}_{T}$ is in $M_{s}^{1}\left(\mathbf{R}^{N}\right)$ for all $s$, we have that $\left(\left\langle\mathbf{g}_{T}, \pi(h) \mathbf{g}_{T}\right\rangle\right)_{h \in D}$ is in $\ell_{s}^{1}(D)$ and ${ }_{D}\left\langle\mathbf{g}_{T}, \mathbf{g}_{T}\right\rangle$ defines an element of $C_{1}^{s}(D, \alpha)$.

A natural interpretation of the quantum theta functions studied in [43-45] comes from the study of the Gabor system $\mathbf{G}\left(\mathbf{g}_{T}, D\right)$ for a general Gaussian $\mathbf{g}_{T}$ and an embedded lattice $D$ of $\mathbf{R}^{2 N}$. The Gabor frame operator $S_{\mathbf{g}_{T}, D}$ looks as follows

$$
S_{\mathbf{g}_{T}, D} \mathbf{f}=\sum_{h \in D}\left\langle\mathbf{f}, \pi(h) \mathbf{g}_{T}\right\rangle \pi(h) \mathbf{g}_{T},
$$

for $\mathbf{f}$ in $L_{2}\left(\mathbf{R}^{N}\right)$. If we consider the Gabor frame operator on $M_{1}^{S}\left(\mathbf{R}^{N}\right)$, then the Janssen representation of $S_{\mathbf{g}_{T}, D}$ exists and turns out to be a quantum theta function:

$$
S_{\mathbf{g}_{T}, D}=\frac{1}{\operatorname{vol}(D)} \sum_{h^{!} \in D^{!}}\left\langle\mathbf{g}_{T}, \pi\left(h^{!}\right) \mathbf{g}_{T}\right\rangle \pi\left(h^{!}\right) .
$$

By Lemma 6.1 the Janssen representation can be rewritten as

$$
S_{\mathbf{g}_{T}, D}=\frac{1}{\operatorname{vol}(D)} \sum_{h^{!} \in D^{!}} \mathrm{e}^{-\pi H_{T}\left(h^{!}, h^{!}\right)} \pi\left(h^{!}\right) .
$$


Therefore, the superposition of time-frequency shifts in the preceding equation

$$
\sum_{h^{\prime} \in D^{!}} \mathrm{e}^{-\pi H_{T}\left(h^{!}, h^{!}\right)} \pi\left(h^{!}\right)
$$

is an element of $C_{1}^{s}\left(D^{!}, \bar{\alpha}^{!}\right)$for each $s$ and consequently of $C^{\infty}\left(D^{!}, \bar{\alpha}^{!}\right)$. In [46] the operator of (6.9) was denoted by $\Theta_{D^{\prime}}$ and it was noted that $\Theta_{D^{\prime}}$ is a quantum theta function in $C_{1}^{s}\left(D^{!}, \alpha\right)$ and consequently in $C^{\infty}\left(D^{!}, \bar{\alpha}^{!}\right)$. More explicitely, $\Theta_{D^{!}}$satisfies the following functional equations:

$$
\forall h^{!} \in D^{!}, C_{h^{!}} \pi_{h !} x_{h^{!}}^{*}\left(\Theta_{D^{!}}\right)=\Theta_{D^{!}},
$$

where

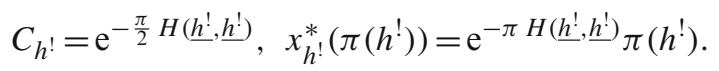

In the Model $I I_{T}$ the quantum theta function $\Theta_{D}$ ! becomes

$$
\Theta_{D} \mathbf{1}(x)=\sum_{h ! \in D} \mathrm{e}^{-\pi H\left(\underline{h}, \underline{h^{!}}\right)-\pi H\left(\underline{x}, \underline{h^{!}}\right)}
$$

where $\mathbf{1}$ is the vacuum vector in the model $\mathrm{II}_{T}$ represented by the function identically equal to 1 .

The function $\Theta_{D} ! \mathbf{1}$ is complex conjugate to the classical theta function corresponding to a principal polarization of the complex torus $\mathbf{C}^{g} / D^{!}$. Notice that this complex torus is embedded into (the space of points of) the algebraic torus $T\left(D^{!}, 1\right)(\mathbf{C})=\operatorname{Hom}\left(D^{!}, \mathbf{C}^{*}\right)$ as its compact subtorus $\operatorname{Hom}\left(D^{!}, \mathbf{C}_{1}^{*}\right)$, see [47].

Moreover, the functional equation for quantum thetas in [46] is an expression for the Janssen representation of $S_{\mathbf{g}_{T}, D} \mathbf{f}$ for $\mathbf{f}=\mathbf{g}_{T}$ :

$$
\sum_{h \in D} \mathrm{e}^{-\pi H(\underline{h}, \underline{h})-\pi H(\underline{x}, \underline{h})}=\frac{1}{\operatorname{vol}(D)} \sum_{h^{\prime} \in D^{!}} \mathrm{e}^{-\pi H\left(\underline{h^{\prime}}, \underline{h^{\prime}}\right)-\pi H\left(\underline{x}, \underline{h^{\prime}}\right)} .
$$

as functions of $x \in \mathbf{R}^{2 N}$.

Since the Janssen representation is a consequence of FIGA one may also consider FIGA for $\mathbf{f}_{1}=\mathbf{f}_{2}=\mathbf{g}_{1}=\mathbf{g}_{2}=\mathbf{g}_{T}$ as a functional equation for quantum thetas. In the case when $N=1$ and $g_{T}$ is the standard Gaussian, Schempp already noted in [61] that this kind of identities are linked with theta functions.

Note that the quantum thetas $\Theta_{D}$ in [46] are defined by ${ }_{D}\left\langle\mathbf{f}_{T}, \mathbf{f}_{T}\right\rangle$, where $\mathbf{f}_{T}$ is our $\mathbf{g}_{i T}$.

We close this section with a few words on the case $D=a \mathbf{Z} \times b \mathbf{Z}$. In this case $H_{T}(z, w)=z \bar{w}$ for $w, z \in \mathbf{C}$. Consequently $\mathbf{g}_{T}$ is just the standard Gaussian $\mathbf{g}_{0}(x)=\mathrm{e}^{-\pi x^{2}}$. Therefore the quantum thetas $\Theta_{a \mathbf{Z}} \times b \mathbf{Z}$ are related to the Gabor systems $\mathbf{G}\left(\mathbf{g}_{0}, a \mathbf{Z} \times b \mathbf{Z}\right)$. A deep result of Lyubarski and Seip obtained independely in [41,62] says that $\mathbf{G}\left(\mathrm{g}_{0}, a \mathbf{Z} \times b \mathbf{Z}\right)$ is a Gabor frame for $L_{2}\left(\mathbf{R}^{N}\right)$ if and only if $a b<1$. An important result of Feichtinger and Gröchenig [18] asserts that Gabor frames 
$\mathbf{G}(g, D)$ for $L_{2}\left(\mathbf{R}^{N}\right)$ with $\mathbf{g}$ in $M_{1}^{s}\left(\mathbf{R}^{N}\right)$ or $\mathcal{S}\left(\mathbf{R}^{N}\right)$ are Banach frames for the class of modulation spaces $M_{p, q}^{m}\left(\mathbf{R}^{N}\right)$. Therefore $\mathbf{G}\left(\mathbf{g}_{0}, a \mathbf{Z} \times b \mathbf{Z}\right)$ is a tight Banach frame for $M_{p, q}^{m}\left(\mathbf{R}^{N}\right)$ if and only if $a b<1$, i.e.

$$
\|\mathbf{f}\|_{M_{p, q}^{m}}=\left(\sum_{l \in \mathbf{Z}}\left(\sum_{k \in \mathbf{Z}}\left|\left\langle\mathbf{f}, \pi(a k, b l) \mathbf{g}_{0}\right\rangle\right|^{p}\right)^{q / p}\right)^{1 / q}<\infty
$$

for all $\mathbf{f}$ in $M_{p, q}^{m}\left(\mathbf{R}^{N}\right)$ and $p, q \in[0, \infty]$. We want to emphasize that the preceding equation provides a description in terms of Gabor coefficients of "good" Gabor frames.

Observe that $\mathbf{G}\left(\mathbf{g}_{0}, a \mathbf{Z} \times b \mathbf{Z}\right)$ is a Gabor frame for $L_{2}(\mathbf{R})$ if and only if the Janssen representation of the Gabor frame operator is invertible. Since the Janssen representation of $S_{\mathbf{g}_{0}, a \mathbf{Z} \times b \mathbf{Z}}$ is the quantum theta $\Theta_{\frac{1}{b} \mathbf{Z} \times \frac{1}{a} \mathbf{Z}}$, we are thus able to produce a precise criterium for the invertiblity of the quantum thetas $\Theta_{\frac{1}{b}} \mathbf{Z} \times \frac{1}{a} \mathbf{Z}$. Moreover the spectral invariance of $C_{1}^{s}\left(\frac{1}{b} \mathbf{Z} \times \frac{1}{a} \mathbf{Z}, \alpha\right)$ in $C^{*}\left(\frac{1}{b} \mathbf{Z} \times \frac{1}{a} \mathbf{Z}, \alpha\right)$ implies that $\Theta_{\frac{1}{b}}^{-1} \mathbf{Z} \times \frac{1}{a} \mathbf{Z}$ is in $C_{1}^{s}\left(\frac{1}{b} \mathbf{Z} \times \frac{1}{a} \mathbf{Z}, \alpha\right)$ if and only if $a b<1$. These observations provide a new approach to the projections in [48] and in addition clarifies the connection between quantum thetas and these projections in quantum tori.

PROPOSITION 6.3. The quantum theta $\Theta_{\frac{1}{b} \mathbf{Z} \times \frac{1}{a} \mathbf{Z}}$ is invertible if and only if $a b<1$.

The construction of projections in higher dimensional quantum tori using Gabor analysis will be addressed by one of us in a subsequent publication. The Gabor systems $\mathbf{G}\left(\mathbf{g}_{T}, D\right)$ will also be studied in more detail.

\section{Acknowledgements}

Large parts of this manuscript were written during a stay of F. Luef at the Max Planck Institute for Mathematics at Bonn. F. Luef would like to express his gratitude for hospitality and excellent working conditions. In addition F. Luef acknowledges the support from the EU-project MEXT-CT-2004-517154 and the Marie Curie Outgoing Fellowship PIOF 220464.

Open Access This article is distributed under the terms of the Creative Commons Attribution Noncommercial License which permits any noncommercial use, distribution, and reproduction in any medium, provided the original author(s) and source are credited.

\section{References}

1. Bastiaans, M.J.: Wigner distribution function and its application to first-order optics. J. Opt. Soc. Am. 69(12), 1710-1716 (1979) 
2. Boca, F.: Projections in rotation algebras and theta functions. Commun. Math. Phys. 202, 325-357 (1999)

3. Cartier, P.: Quantum mechanical commutation relations and theta functions. In: Algebraic Groups and Discontinuous Subgroups (Proc. Sympos. Pure Math., Boulder, Colo., 1965), pp. 361-383. Amer. Math. Soc., Providence (1966)

4. Young, E.C., Kim, H.: Theta vectors and quantum theta functions. J. Phys. A 38(19), 4255-4263 (2005)

5. Connes, A.: $C^{*}$-algèbres et géométrie différentielle. C. R. Acad. Sci. Paris Sér. AB 290(13), A599-A604 (1980)

6. Connes, A.: An analogue of the Thom isomorphism for crossed products of a $C^{*}$ algebra by an action of R. Adv. Math. 39(1), 31-55 (1981)

7. Connes, A.: Noncommutative Geometry. Academic Press, London (1994)

8. Daubechies, I., Grossmann, A., Meyer, Y.: Painless nonorthogonal expansions. J. Math. Phys. 27(5), 1271-1283 (1986)

9. Daubechies, I.: The wavelet transform, time-frequency localization and signal analysis. IEEE Trans. Inf. Theory 36(5), 961-1005 (1990)

10. Daubechies, I., Landau, H.J., Landau, Z.: Gabor time-frequency lattices and the Wexler-Raz identity. J. Fourier Anal. Appl. 1(4), 437-478 (1990)

11. Duffin, R.J., Schaeffer, A.C.: A class of nonharmonic Fourier series. Trans. Am. Math. Soc. 72, 341-366 (1952)

12. Ee, C.-Y., Kim, H.: Quantum thetas on noncommutative $\mathbb{T}^{d}$ with general embeddings. e-print (math-ph/0709.2483)

13. Farden, D.C., Scharf, L.L.: A unified framework for the Sussman, Moyal, and Janssen formulas. IEEE Sig. Process. Mag. 124, 124-125 (2006)

14. Feichtinger, H.G.: On a new Segal algebra. Monatsh. Math. 92, 269-289 (1981)

15. Feichtinger, H.G.: Modulation spaces on locally compact Abelian groups. Technical Report, January 1983

16. Feichtinger, H.G.: Modulation spaces: looking back and ahead. Sampl. Theory Sig. Image Process. 5(2), 109-140 (2006)

17. Feichtinger, H.G., Gröchenig, K.: Banach spaces related to integrable group representations and their atomic decompositions, I. J. Funct. Anal. 86, 307-340 (1989)

18. Feichtinger, H.G., Gröchenig, K.: Gabor frames and time-frequency analysis of distributions. J. Funct. Anal. 146(2), 464-495 (1997)

19. Feichtinger, H.G., Kozek, W.: Quantization of TF lattice-invariant operators on elementary LCA groups. In: Feichtinger, H., Strohmer, T. (eds.) Gabor Analysis and Algorithms. Theory and Applications., Applied and Numerical Harmonic Analysis, pp. 233-266, 452-488. Birkhäuser, Boston (1998)

20. Feichtinger, H.G., Luef, F.: Wiener amalgam spaces for the fundamental identity of Gabor analysis. Collect. Math., 57, 233-253 (2006) e-Print (math.FA/0503364)

21. Folland, G.B.: Harmonic Analysis in Phase Space. Princeton University Press, Princeton (1989)

22. Frank, M., Larson, D.R.: Frames in Hilbert $C^{*}$-modules and $C^{*}$-algebras. J. Oper. Theory 48, 273-314 (2002)

23. Gabor, D.: Theory of communication. J. IEE 93(26), 429-457 (1946)

24. deGosson, M.: Symplectic geometry and quantum mechanics. Operator Theory: Advances and Applications. Advances in Partial Differential Equations, vol. 166. Birkhäuser, Boston (2006)

25. Gröchenig, K.: Aspects of Gabor analysis on locally compact abelian groups. In: Feichtinger, H., Strohmer, T. (eds.) Gabor analysis and algorithms: Theory and Applications, pp. 211-231. Birkhäuser, Boston (1998) 
26. Gröchenig, K.: Foundations of time-frequency analysis. Appl. Numer. Harmon. Anal. Birkhäuser, Boston (2001)

27. Gröchenig, K., Leinert, M.: Wiener's lemma for twisted convolution and Gabor frames. J. Am. Math. Soc. 17(1), 1-18 (2004)

28. Gröchenig, K., Luef, F.: The topological stable rank of projective modules over noncommutative tori (2008, preprint)

29. Howe, R.: On the role of the Heisenberg group in harmonic analysis. Bull. Am. Math. Soc. 3(2), 821-843 (1980)

30. Igusa, J.-i.: Theta functions. Die Grundlehren der mathematischen Wissenschaften, Band 194. Springer, Heidelberg (1972)

31. Janssen, A.J.E.M.: Gabor representation of generalized functions. J. Math. Anal. Appl. 83, 377-394 (1981)

32. Janssen, A.J.E.M.: Weighted Wigner distributions vanishing on lattices. J. Math. Anal. Appl. 80, 156-167 (1981)

33. Janssen, A.J.E.M.: Bargmann transform, Zak transform, and coherent states. J. Math. Phys. 23(5), 720-731 (1982)

34. Janssen, A.J.E.M.: Duality and biorthogonality for Weyl-Heisenberg frames. J. Fourier Anal. Appl. 1(4), 403-436 (1995)

35. Kontsevich, M.: Deformation quantization of algebraic varieties. e-Print (math.AG/0106006)

36. Littlejohn, R.G.: The semiclassical evolution of wave packets. Phys. Rep. 138(4-5), 193-291 (1986)

37. Luef, F.: Gabor Analysis meets Noncommutative Geometry. Ph.D. Thesis, University of Vienna, November 2005

38. Luef, F.: On spectral invariance of non-commutative tori. In: Operator theory, operator algebras, and applications, vol. 414, pp. 131-146. American Mathematical Society (2006) e-Print (math.OA/0603139)

39. Luef, F.: Gabor analysis, noncommutative tori and Feichtinger's algebra. In: Gabor and Wavelet Frames. IMS Lecture Notes Series, vol. 10, pp. 77-106. World Sci. Pub (2007) e-Print (math.FA/0504146)

40. Luef, F.: Projective modules over non-communtative tor are multi-window Gabor frames for modulation spaces. e-Print (math.OA/0807.3170)

41. Lyubarskij, Y.I.: Frames in the Bargmann space of entire functions. In Entire and subharmonic functions. Adv. Sov. Math., vol. 11, pp. 167-180. American Mathematical Society (AMS), Providence (1992)

42. Manin, Yu.: Quantized theta-functions. In: Common Trends in Mathematics and Quantum Field Theories (Kyoto, 1990), Progress of Theor. Phys. Supplement, vol. 102, pp. 219-228 (1990)

43. Manin, Yu.: Mirror symmetry and quantization of abelian varieties. In: Faber, C., et al. (eds.) Moduli of Abelian Varieties. Progress in Math., vol. 195, pp. 231-254. Birkhäuser, Boston (2001) e-print (math.AG/0005143)

44. Manin, Yu.: Theta functions, quantum tori and Heisenberg groups. Lett. Math. Phys. 56(3), 295-320 (2001) e-Print (math.AG/001119c)

45. Manin, Yu.: Real multiplication and noncommutative geometry (ein Alterstraum). In: The legacy of Niels Henrik Abel, pp. 685-727. Springer, Berlin (2004) e-Print (math.AG/0202109)

46. Manin, Yu.: Functional equations for quantum theta functions. Publ. Res. Inst. Math. Sci. 40(3), 605-624 (2004) e-print (math.QA/0307393)

47. Mumford, D., Nori, M., Norman, P.: Tata Lectures on Theta III. Progress Math., vol. 97. Birkhäuser, Boston (1991) 
48. Polishchuk, A.: Abelian varieties, theta functions and the Fourier transform. Cambridge Tracts in Mathematics, vol. 153. Cambridge University Press, Cambridge (2003)

49. Raeburn I., Williams, D.P.: Morita Equivalence and Continuous-trace $C^{*}$-algebras. American Mathematical Society (AMS), RI (1998)

50. Reiter, M.: Über den Satz von Weil-Cartier. Mh. Math. 86, 13-62 (1978)

51. Reiter, M.: Theta functions and symplectic groups. Mh. Math. 97, 219-232 (1984)

52. Reiter, H.: Metaplectic Groups and Segal Algebras. Springer, Berlin (1989)

53. Rieffel, M.A.: $C^{*}$-algebras associated with irrational rotations. Pac. J. Math. 93, 415-429 (1981)

54. Rieffel, M.A.: The cancellation theorem for projective modules over irrational rotation $C^{*}$-algebras. Proc. Lond. Math. Soc. III. Ser. 47, 285-302 (1983)

55. Rieffel, M.A.: Projective modules over higher-dimensional non-commutative tori. Can. J. Math. XL(2), 257-338 (1988)

56. Rieffel, M.A.: Non-commutative tori-a case study of non-commutative differential manifolds. Cont. Math. 105, 191-211 (1990)

57. Rieffel, M.A., Schwarz, A.: Morita equivalence of multidimensional non-commutative tori. Int. J. Math. 10, 289-299 (1999) e-print (math.QA/9803057)

58. Rosenberg, J.: Noncommutative variations on Laplace's equation (2008) e-Print (math.OA/0802.4033)

59. Ron, A., Shen, Z.: Weyl-Heisenberg frames and Riesz bases in $L_{2}\left(\mathbf{R}^{d}\right)$. Duke Math. J. 89(2), 237-282 (1997)

60. Schwarz, A.: Theta-functions on non-commutative tori. Lett. Math. Phys. 58(1), 81-90 (2001) e-Print (math.QA/0107186)

61. Schempp, W.: Radar ambiguity functions, the Heisenberg group, and holomorphictheta series. Proc. Am. Math. Soc. 92, 103-110 (1984)

62. Seip, K.: Density theorems for sampling and interpolation in the Bargmann-Fock space. I. J. Reine Angew. Math. 429, 91-106 (1992)

63. Sussman, S.M.: Least square synthesis of radar ambiguity functions. IRE Trans. Inf. Theory 8, 246-254 (1962)

64. Vlasenko, M.: The graded ring of quantum theta functions for noncommutative torus with real multiplication. Int. Math. Res. Not. 1-19 (2006)

65. Weil, A.: Sur certains groupes d'opérateurs unitaires. Acta Math. 111, 143-211 (1964) 